\title{
Viscous sheet retraction
}

\author{
NIKOS SAVVA† AND JOHN W. M. BUSH† \\ Department of Mathematics, Massachusetts Institute of Technology, \\ Cambridge, MA 02139, USA
}

(Received 11 December 2007 and in revised form 19 November 2008)

We present the results of a combined theoretical and numerical investigation of the rim-driven retraction of flat fluid sheets in both planar and circular geometries. Particular attention is given to the influence of the fluid viscosity on the evolution of the sheet and its bounding rim. In both geometries, after a transient that depends on the sheet viscosity and geometry, the film edge eventually attains the TaylorCulick speed predicted on the basis of inviscid theory. The emergence of this result in the viscous limit is rationalized by consideration of both momentum and energy arguments. We first consider the planar geometry considered by Brenner \& Gueyffier (Phys. Fluids, vol. 11, 1999, p. 737) and deduce new analytical expressions for the speed of the film edge at the onset of rupture and the evolution of the maximum film thickness for viscous films. In order to consider the expansion of a circular hole, we develop an appropriate lubrication model that predicts the form of the early stage dynamics of film rupture. Simulations of a broad range of flow parameters confirm the importance of geometry on the dynamics, verifying the exponential hole growth reported in early experimental studies. We demonstrate the sensitivity of the initial retraction speed on the film profile, and so suggest that the anomalous rate of retraction reported in these experiments may be attributed in part to geometric details of the puncture process.

\section{Introduction}

Sheet retraction arises in a wide range of physical settings, ranging from fuel injectors to foams in the food industry to biological membranes (for an overview see de Gennes, Brochart-Wyart \& Quéré 2003). The disintegration of fluid sheets is of primary importance in the context of fluid fragmentation or atomization (Lefebvre 1989; Bayvel \& Orzechowski 1993; Villermaux 2007). Commonly, such atomization processes involve a cascade from fluid volumes to sheets to filaments to droplets, a route that depends critically on the dynamics and stability of fluid sheets and their bounding rims. Depending on the application at hand, film rupture can be either desirable, as in spray formation (e.g. Pomeau \& Villermaux 2006), or undesirable, as in curtain coating (e.g. Miyamoto \& Katagiri 1997).

The initial observations of soap film rupture were reported by Dupré (1867) and Rayleigh (1891). Their studies motivated the experimental work of Ranz (1950), who observed that, following puncture, the film recedes under the influence of surface tension at a constant speed, and that fluid accumulates in a roughly circular rim as

$\dagger$ Present address: Department of Chemical Engineering, Imperial College London, London SW7 2AZ, UK.

$\ddagger$ E-mail address for correspondence: bush@math.mit.edu 


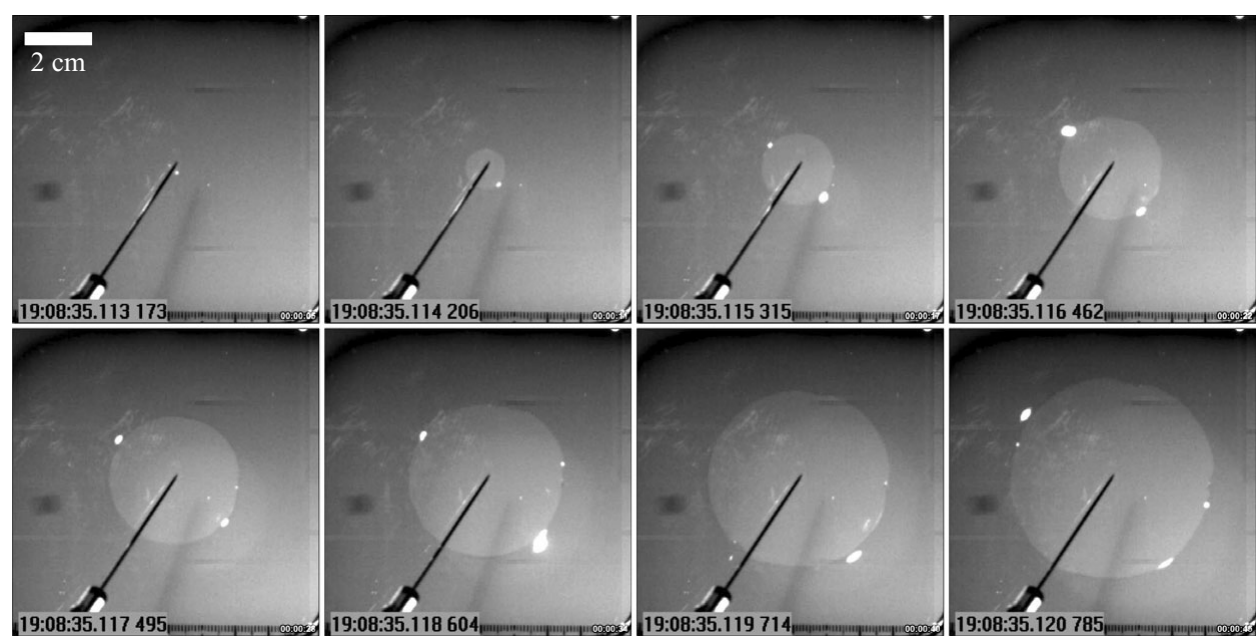

FIgURE 1. The bursting of a soap film. Times are indicated in each frame. The sequence lasts approximately $7.6 \mathrm{~ms}$.

it is drawn away from the point of puncture (see figure 1). Dupré (1867) incorrectly deduced the retraction speed of the film, based on the erroneous assumption that the surface energy lost due to retraction is converted purely into kinetic energy

$$
U=\sqrt{\frac{4 \gamma}{\rho H}}=\sqrt{2} u_{c},
$$

where $\rho, \gamma, H$ correspond to the density, surface tension and thickness of the film, respectively. Taylor (1959) and Culick (1960) independently corrected Duprés calculation using an argument based on the conservation of momentum of the film and found that the retraction speed $u_{c}$ was a factor of $\sqrt{2}$ less than that predicted by Dupré, as will be reviewed in $\$ 2.1$.

McEntee \& Mysels (1969) confirmed experimentally the Culick-Taylor theory for soap films of thickness greater than $0.1 \mu \mathrm{m}$. Keller (1983) extended the previous work of Taylor (1959) and Culick (1960) by considering sheets of non-uniform thickness and Keller \& Miksis (1983) considered time-dependent inviscid potential flows in which a wedge-shaped initial free surface profile admits self-similar solutions. In $\S 2.1$ we shall demonstrate why $u_{c}$ is achieved even in the viscous limit, where there is fluid motion upstream of the tip.

While the bulk of experiments on retracting fluid sheets have been conducted with water sheets or soap films in air, Debrégeas, Martin \& Brochard-Wyart (1995) and Debrégeas, de Gennes \& Brochard-Wyart (1998) presented a series of experiments using films with viscosity of the order of one million times that of water. They used polymer polydimethylsiloxane (PDMS) films obtained by dipping a ring into an isopentane solution of the polymer. After lifting the ring from the solution, the solvent evaporated, leaving behind a suspended film of pure PDMS with thickness of the order of $10-50 \mu \mathrm{m}$, much thicker than a typical soap film (of characteristic thickness $\sim 1 \mu \mathrm{m}$ ). Accurate measurements of the retraction process revealed behaviour that was markedly different from that predicted by inviscid theory. In particular, they found that the fluid no longer collects in a rim as it retracts. Moreover, the rim does 
not retract at a constant speed; rather, the hole radius grows exponentially as

$$
r_{0_{\text {exp }}}(t)=R_{0} \exp \left(\frac{t}{1.4 \tau}\right),
$$

where $\tau=\mu H / 2 \gamma$, with $R_{0}$ being the initial hole size and $\mu$ the film's dynamic viscosity. The exponential hole growth was also supported by a simple theoretical argument, in which surface energy released during retraction was equated to the energy dissipated through the action of viscosity, that predicted:

$$
r_{0}(t)=R_{0} \exp \left(\frac{t}{2 \tau}\right) .
$$

While Debrégeas et al. (1995) did not consider viscoelastic effects in deriving (1.3), they did suggest that the exponential behaviour may be due in part to the viscoelasticity of the films. However, the typical shear rate of the PDMS films was below those at which non-Newtonian behaviour sets in (e.g. Deyrail et al. 2007). In the subsequent experimental studies of Dalnoki-Veress et al. (1999) and Roth et al. (2005), film rupture was used as a means to measure the viscosity of molten polystyrene films with viscosities of the order of $10^{12}$ times that of water. Dalnoki-Veress et al. (1999) probed the nonlinear viscoelastic regime, where it was found that the viscous time scale $\tau$ decreased markedly with decreasing film thickness. This effect was attributed to the reduction in film viscosity caused by the large shear strain rates caused by the hole expansion. Roth et al. (2005) found an initial transient regime where the expansion of the hole was faster than exponential; fitting to the data was achieved by introducing a time-dependent viscosity.

Following the work of Debrégeas et al. (1995), Brenner \& Gueyffier (1999) studied the retraction of a two-dimensional planar sheet numerically using a one-dimensional Trouton-type lubrication model. They were able to identify three distinct regimes depending on the Ohnesorge number, defined as

$$
O h=\frac{\mu}{\sqrt{2 H \rho \gamma}}
$$

that expresses the relative importance of viscous resistance to surface tension forces (see figure 2). In the low $O h$ regime $(O h<0.1)$, they found that capillary wave disturbances are generated ahead of the retracting rim. As the Ohnesorge number is increased, the capillary waves disappear and the rim diffuses in towards the bulk of the sheet. Finally in the high $O h$ regime $(O h \gg 10)$, they found that no rim forms at all, in accord with Debrégeas' observations. In all cases considered, the sheet edge was found to approach the Culick-Taylor speed in the long time limit.

The retraction time scales are different in the high and low $\mathrm{Oh}$ regimes. Since numerical simulations indicate that the characteristic speed $u_{c}$ is independent of $\mathrm{Oh}$, the length scale prescribes the characteristic retraction time. Naturally, this length scale is a measure of the distance from the tip over which the film is disturbed, and depends on the relative importance of viscosity to surface tension, as quantified through $\mathrm{Oh}$. In the low $\mathrm{Oh}$ regime, the motion is primarily concentrated near the tip, so that the characteristic length scale is prescribed by $H$. This implies that the characteristic time scale is

$$
\tau_{i n v}=\frac{H}{u_{c}}=\sqrt{\frac{\rho H^{3}}{2 \gamma}},
$$

which is of the order of microseconds for the soap films in the experiments of Ranz (1950). Conversely, in the viscous, high Oh limit, a larger portion of the film 


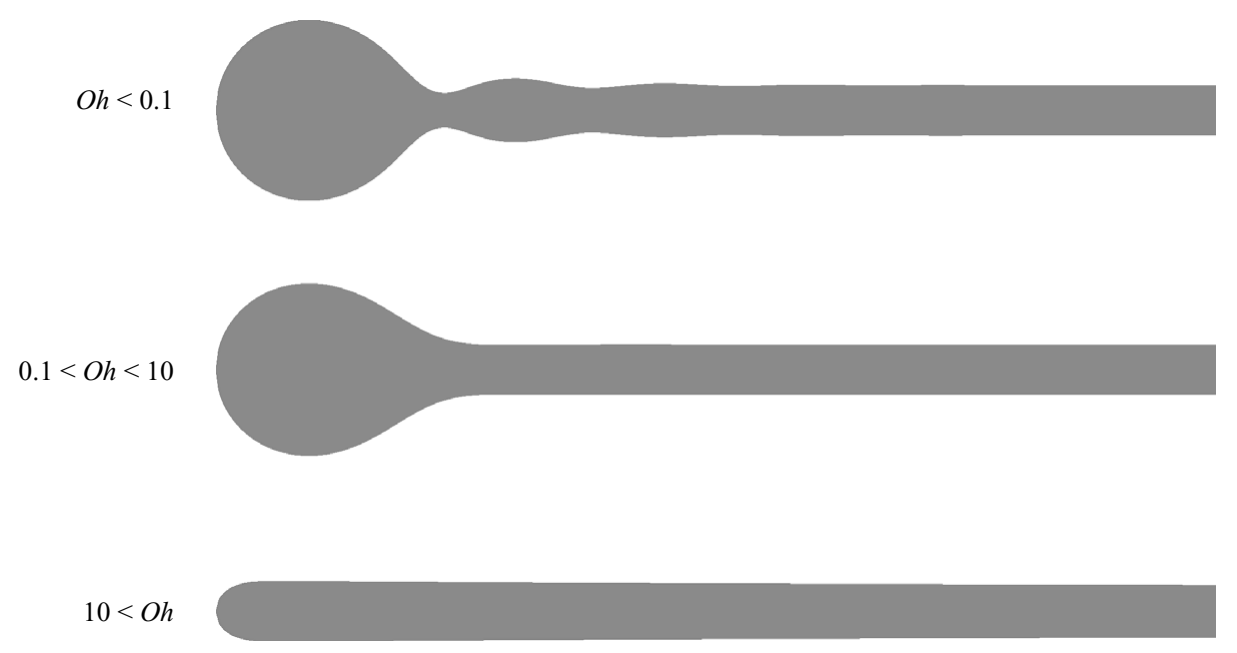

FIGURE 2. Schematic illustration of the three distinct regimes obtained for a retracting sheet by Brenner \& Gueyffier (1999). As Oh progressively increases, the capillary waves and then the rim disappear through the action of viscosity.

is accelerated by the unbalanced surface tension force near the tip and the flow is characterized by a length scale $O h H$ equivalent to the Stokes length $\mu / \rho u_{c}$ identified by Brenner \& Gueyffier (1999). Hence the characteristic retraction time is given by

$$
\tau_{v i s}=\frac{O h H}{u_{c}}=\frac{\mu H}{2 \gamma} \text {. }
$$

This time was of the order of seconds for the PDMS films used in Debrégeas et al. (1995) and hours for the polystyrene films used in the experiments of Roth et al. (2005).

Following the work of Brenner \& Gueyffier (1999), two-dimensional simulations of planar sheet retraction were presented by Song \& Tryggvason (1999), who took into account the effect of the ambient fluid. They found that even when the ratio of ambient to sheet viscosity is $O(0.1)$, the influence of the ambient fluid is minor. Their results however were somewhat limited due to the short extent of the fluid sheet, and no conclusion could be drawn concerning the dynamics in the long time limit. More recently, Sünderhauf, Raszillier \& Durst (2002) performed two-dimensional simulations of the Navier-Stokes equations, but neglected the ambient fluid on the basis of the prior work of Song \& Tryggvason (1999). They focused primarily on exploring the acceleration phase of the film edge towards the terminal Taylor-Culick speed and provided some insights into the stability of falling liquid sheets. Critically, these previous works were not able to capture the exponential regime observed by Debrégeas et al. (1995), a shortcoming that, as suggested by Brenner \& Gueyffier (1999) and demonstrated here, can be attributed to the planar geometry considered.

We here elaborate on the retraction dynamics of flat sheets by building upon the work of Brenner \& Gueyffier (1999); specifically, we clarify the effects of viscosity and geometry by solving the appropriate Trouton-type model for both planar and circular geometries. In $\S 2$, we further explore the model of Brenner \& Gueyffier (1999) for planar sheets in order to obtain several new insights. In $\S 2.1$ we present the conservation laws and use them to clarify why (2.3) adequately describes flow with viscous dissipation, despite the fact that viscosity was neglected in its derivation. In 


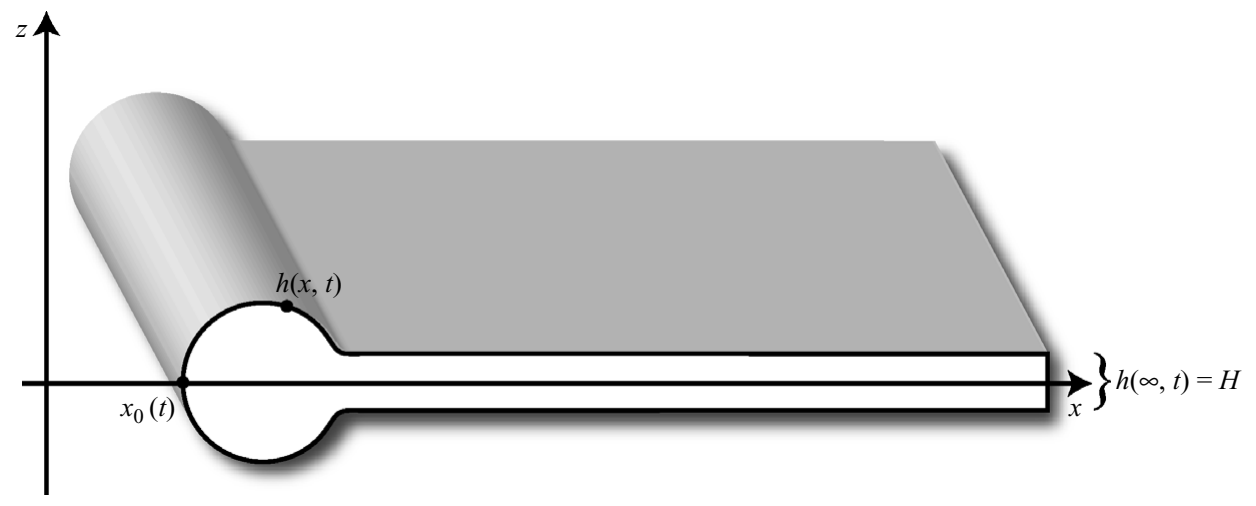

FIGURE 3. Planar sheet geometry. The sheet retracts from left to right under the influence of the capillary force acting at its edge.

$\S 2.2$, we calculate an analytic expression for the hole growth during the early stages of retraction and the maximum film thickness in the high $O h$ limit. In $\S 3$, we develop a new theoretical model that allows us to investigate the expansion of a circular hole for arbitrary Ohnesorge number. By examining the lubrication equations in the high $O h$ limit, we demonstrate that the exponential hole growth in the high $O h$ limit can be deduced directly from the lubrication equations, thus providing an alternative derivation to the energy argument of Debrégeas et al. (1995). Our model is solved numerically to elucidate the effects of viscosity, geometry and initial conditions. We conclude in $\S 4$ with a discussion of the potential importance of three-dimensional and other effects neglected in our model.

\section{Planar sheets}

We proceed by considering the model of Brenner \& Gueyffier (1999) for the retraction of a planar semi-infinite viscous sheet under the influence of surface tension (figure 3). For a planar sheet of thickness $h(x, t)$ moving under the influence of surface tension forces along the $x$-axis with speed $u(x, t)$

$$
\begin{aligned}
u_{t}+u u_{x} & =\frac{4 \mu}{\rho h}\left(h u_{x}\right)_{x}+\frac{\gamma}{\rho} \kappa_{x}, \\
h_{t}+(h u)_{x} & =0,
\end{aligned}
$$

where $\kappa(x, t)$ corresponds to the curvature of the film profile,

$$
\kappa=\frac{(1 / 2) h_{x x}}{\left(1+(1 / 4) h_{x}^{2}\right)^{3 / 2}} .
$$

Experimental study of the retraction of a planar film is impractical owing to unavoidable edge effects and the difficulties inherent in producing a perfectly linear rupture. For films bound between two parallel wires, edge effects can become dominant and the retraction rate significantly reduced (Chepushtanova \& Kliakhandler 2007). Nevertheless the planar geometry is governed by relatively simple equations that we show yield analytic solutions for the early expansion. Moreover, it yields physical insight that shall guide us in modelling the more experimentally feasible circular geometry. 


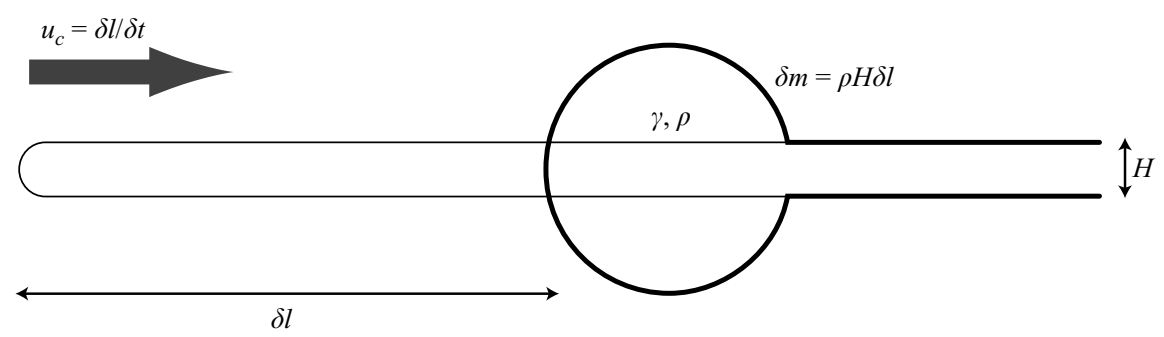

FIGURE 4. Retraction of a planar film. In time $\delta t$, the tip of the film moves by a distance $\delta l=U_{c} \delta t$. The mass of the fluid (per unit length) accumulated in the rim during that time is $\delta m=\rho H \delta l$.

\subsection{Conservation laws}

Taylor (1959) and Culick (1960) deduced the constant speed of retraction by assuming that the film preceding the rim is at rest and expressing the force balance on the rim as a balance between the rate of change of rim momentum $P_{\text {rim }}$ and the surface tension force exerted on the rim

$$
\frac{\mathrm{d} P_{r i m}}{\mathrm{~d} t}=u_{c} \frac{\mathrm{d} m}{\mathrm{~d} t}=2 \gamma,
$$

where $u_{c}$ is the constant rim speed and $m$ the rim mass per unit length (see figure 4). The rate of change of the rim mass satisfies

$$
\frac{\mathrm{d} m}{\mathrm{~d} t}=\rho H u_{c} .
$$

Henceforth, the constant speed

$$
u_{c}=\sqrt{\frac{2 \gamma}{\rho H}}
$$

found from (2.3) and (2.4) became known to be the Taylor-Culick speed.

To demonstrate that (2.3) arises at arbitrary viscosities, we consider a planar sheet of finite initial length $L$. At any instant, the edge of the sheet is located at $x_{0}(t)$. Imposing the conditions

$$
\begin{aligned}
h\left(x_{0}, 0\right) & =h_{x}(L, t)=h_{x x}(L, t)=0, \\
u(L, t) & =u_{x}(L, t)=0 \text { and } \\
h(L, t) & =H,
\end{aligned}
$$

we can deduce the conservation laws for the mass, momentum and energy. The total mass of the fluid (per unit length) is

$$
m=\rho \int_{x_{0}}^{L} h \mathrm{~d} x .
$$

Mass conservation trivially follows from (2.1b) since

$$
\frac{\mathrm{d} m}{\mathrm{~d} t}=\rho \int_{x_{0}}^{L} h_{t} \mathrm{~d} x=-\left.\rho h u\right|_{x_{0}} ^{L}=0 .
$$

More importantly, an expression for the total sheet momentum,

$$
P_{t o t}=\rho \int_{x_{0}}^{L} h u \mathrm{~d} x,
$$


can be found by multiplying $(2.1 b)$ by $u$ and $(2.1 a)$ by $h$ and adding them together. Thus, we obtain the equation

$$
(\rho h u)_{t}+\left(\rho h u^{2}-4 \mu h u_{x}-\gamma h \kappa-2 \gamma\left(1+\frac{1}{4} h_{x}^{2}\right)^{-1 / 2}\right)_{x}=0 .
$$

Integrating the first term from $x_{0}$ to $L$ gives

$$
\rho \int_{x_{0}}^{L}(h u)_{t} \mathrm{~d} x=\frac{\mathrm{d} P_{t o t}}{\mathrm{~d} t}+\rho u\left(x_{0}\right)^{2} h\left(x_{0}\right)=\frac{\mathrm{d} P_{t o t}}{\mathrm{~d} t} .
$$

Combining this with the integral of the second term from $x_{0}$ to $L$, evaluated by using (2.6), yields

$$
\frac{\mathrm{d} P_{t o t}}{\mathrm{~d} t}=2 \gamma
$$

under the assumption that $h_{x} \rightarrow \infty$ as $x \rightarrow x_{0}$. Hence, we find that even though the dissipation due to viscosity is not directly described by Taylor's and Culick's momentum balance, its inclusion does not alter the veracity of their result. With this simple calculation, we see the role of viscosity in the dynamics of retraction: it affects how the momentum is distributed through the film, but does not affect its terminal speed $u_{c}$. As also pointed out in the two-dimensional numerical calculations of Sünderhauf et al. (2002), in the long time limit, half of the surface energy is converted to kinetic energy, while the other half is ultimately dissipated through the action of viscosity.

To obtain the corresponding energy equation, we multiply $(2.1 b)$ by $u^{2}$ and $(2.1 a)$ by $h u$ and add them, which yields, after some algebra:

$$
\begin{array}{r}
\left(\frac{1}{2} \rho h u^{2}+2 \gamma \sqrt{1+\frac{1}{4} h_{x}^{2}}\right)_{t}+\left(\frac{1}{2} \rho h u^{3}-4 \mu h u u_{x}-\gamma u h \kappa-\frac{(1 / 2) \gamma h_{x} h_{t}}{\left(1+(1 / 4) h_{x}^{2}\right)^{1 / 2}}\right)_{x} \\
=-4 \mu h u_{x}^{2} .
\end{array}
$$

Integration with respect to $x$ and use of the boundary conditions (2.6) yields

$$
\frac{\mathrm{d}}{\mathrm{d} t}\left(E_{k}+E_{\gamma}\right)=D
$$

where we identify

$$
\begin{aligned}
E_{k} & =\frac{1}{2} \rho \int_{x_{0}}^{L} h u^{2} \mathrm{~d} x, \\
E_{\gamma} & =2 \gamma \int_{x_{0}}^{L} \sqrt{1+\frac{1}{4} h_{x}^{2}} \mathrm{~d} x, \\
D & =-4 \mu \int_{x_{0}}^{L} h u_{x}^{2} \mathrm{~d} x,
\end{aligned}
$$

as the total kinetic energy, surface energy and viscous dissipation of the sheet, respectively. This approach reveals why Duprés original argument of balancing surface energy lost and kinetic energy gained by the retracting film predicted an incorrect retraction speed. Culick (1960) argued against the prediction of Dupré (1867) on physical grounds, attributing the discrepancy to the energy lost while the undisturbed film accelerates to the constant Taylor-Culick speed. We now see that the momentum conservation equation yields the correct expression for $u_{c}$, since the 
viscous forces are internal to the film and so do not contribute to the momentum budget (2.12).

\subsection{Early stages of retraction}

We proceed by deducing a new analytic expression that describes the initiation of sheet retraction in the high $O h$ limit. Non-dimensionalizing the equations by

$$
t=\tau_{v i s} t^{*}, x=O h H x^{*}, h=H h^{*} \text { and } u=u_{c} u^{*},
$$

we can write the momentum equation in non-dimensional form as

$$
u_{t^{*}}^{*}+u^{*} u_{x^{*}}^{*}=\frac{4}{h^{*}}\left(h^{*} u_{x^{*}}^{*}\right)_{x^{*}}+\frac{1}{2} O h^{-2} \kappa_{x^{*}}^{*} .
$$

If we assume that the fluid sheet consists of a nearly semi-circular cap followed by a straight edge, there occurs a singularity in the curvature at $x_{s}^{*}=(2 O h)^{-1}$, since at $t^{*}=0$ it satisfies

$$
\kappa^{*}=\left\{\begin{array}{cl}
-2 O h^{2} & 0 \leqslant x^{*}<(2 O h)^{-1} \\
0 & x^{*}>(2 O h)^{-1}
\end{array} .\right.
$$

Therefore, we can approximate $\kappa_{x^{*}}^{*}$ initially by a delta function

$$
\kappa_{x^{*}}^{*}=2 O h^{2} \delta\left(x^{*}-x_{s}^{*}\right)
$$

We further assume that during the initial stages of retraction, the film thickness remains uniform to leading order, $x_{s}^{*}$ is small compared to the axial extent of the film, the delta-function structure of the curvature gradient $\kappa_{x^{*}}^{*}$ is preserved and the fluid contained in the semi-circular cap moves at the tip speed. By neglecting the convective term, we thus reduce the problem to

$$
u_{t}=4 u_{x x}+\delta\left(x-x_{s}\right),
$$

where we have dropped the stars for convenience. These simplifying assumptions are expected to be strictly valid only in the high $\mathrm{Oh}$ regime, where the viscous effects dominate inertia at the onset and resist the shape change of the fluid film, specifically the development of a pronounced rim.

The velocity is assumed to be continuous, which allows us to integrate (2.21) from $x_{s}-\varepsilon$ to $x_{s}+\varepsilon$ for some small $\varepsilon>0$. Taking the limit as $\varepsilon \rightarrow 0$ yields a jump condition for $u_{x}$

$$
\left[u_{x}\right]=-\frac{1}{4} .
$$

Away from the discontinuity at $x_{s}$ (which without loss of generality we set as $x_{s}=0$ ) we are left with the heat equation

$$
u_{t}=4 u_{x x}, x \geqslant 0
$$

which we solve subject to the conditions

$$
u(x, 0)=0, \quad u_{x}(0, t)=-\frac{1}{4}, \quad u(x, t) \rightarrow 0 \quad \text { as } x \rightarrow \infty,
$$

the second of which comes from the jump in $u_{x}$ at $x_{s}$ and is obtained when there are no velocity gradients within the fluid cap. The solution to $(2.23)$ can be obtained by the method of Laplace transforms that yields

$$
u(x, t)=\sqrt{\frac{t}{\pi}} \exp \left(-\frac{x^{2}}{16 t}\right)-\frac{1}{4} x \operatorname{erfc}\left(\frac{x}{4 \sqrt{t}}\right) .
$$




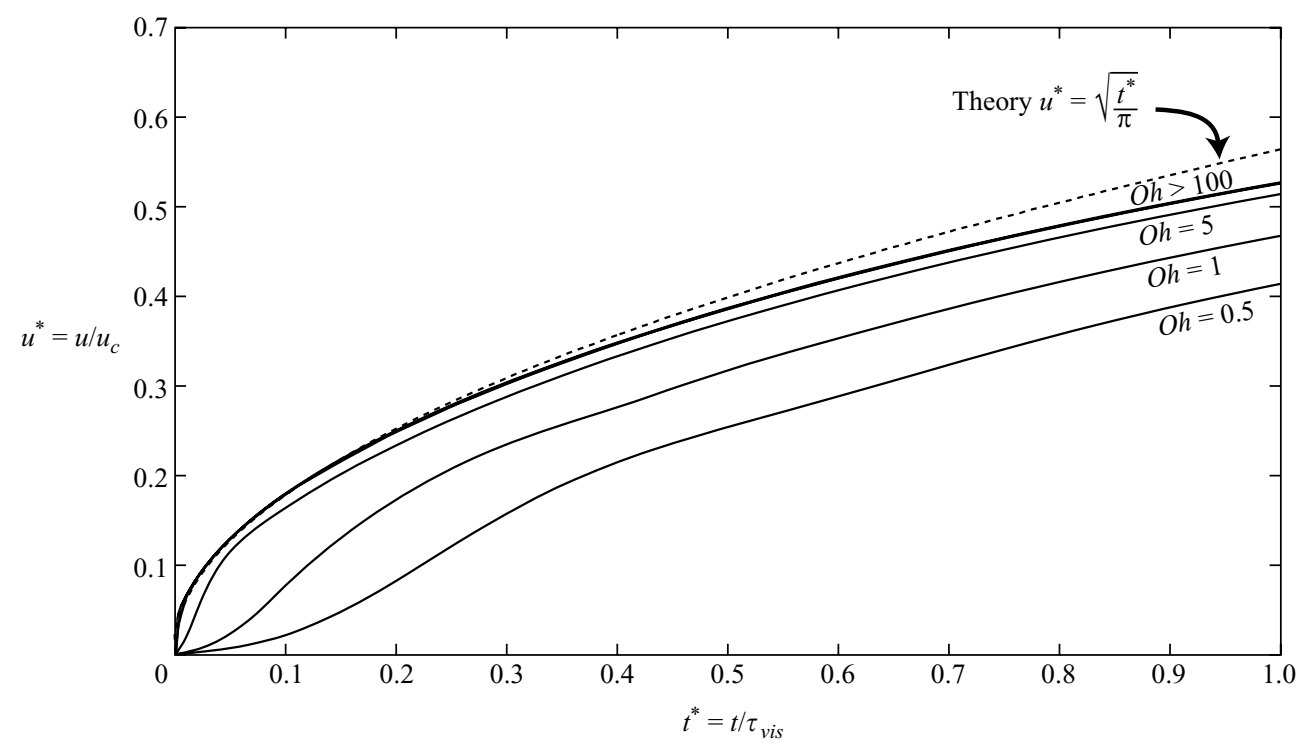

FiguRE 5. Plot of the tip velocity versus time during the early stages of retraction for different Ohnesorge numbers. The plots for $O h=100,500,1000$ and 10000 are indistinguishable. The dotted curve shows the theoretical result $u^{*}=\sqrt{t^{*} / \pi}$, which is in good agreement with numerics up to a time $t \approx 0.4 \tau_{v i s}$, where $\tau_{v i s}=\mu H / 2 \gamma$.

In dimensional variables, the tip speed and displacement may be expressed as

$$
u(0, t)=u_{c} \sqrt{\frac{t}{\pi \tau_{v i s}}}, \quad x_{0}=\frac{2}{3 \sqrt{\pi}}\left(\frac{t}{\tau_{v i s}}\right)^{3 / 2} H .
$$

Solving (2.1) together with conditions (2.6) in a similar manner as described in the Appendix for the axisymmetric case, we show in figure 5 the evolution of the tip speed for various Ohnesorge numbers that verifies the validity of our calculation for short times. This result supports the suggestion of Brenner \& Gueyffier (1999) that the geometry plays an important role in the retraction dynamics. The edge of a planar film initially recedes with a displacement that scales as $t^{3 / 2}$ while in the experiments reported in Debrégeas et al. (1995), Dalnoki-Veress et al. (1999) and Roth et al. (2005), the initial retraction of a circular hole follows an exponential law.

Despite our simplifying assumptions, the analytical solution obtained matches perfectly the numerical simulations at early times $\left(t<0.2 \tau_{v i s}\right)$. At later times and for $O h>50$, the agreement is quite good with an error of less than $6 \%$ at $t \approx \tau_{v i s}$. Beyond that time, a number of neglected effects become significant, such as $h_{x}$ and the convective term, thus rendering our approximation invalid. In figure 6 , we plot the evolution of the tip speed for various $O h$. We see that for films of $O h>100$, the tip speeds converge. This behaviour could be anticipated from (2.21), where Oh does not appear.

\subsection{Maximum film thickness}

In the high $\mathrm{Oh}$ limit, no visible rim forms as the sheet retracts; therefore, by mass conservation, the film thickness must increase with time. To find the evolution of the film thickness $h_{m}(t)$, we examine (2.18). We assume that the semi-circular cap is 


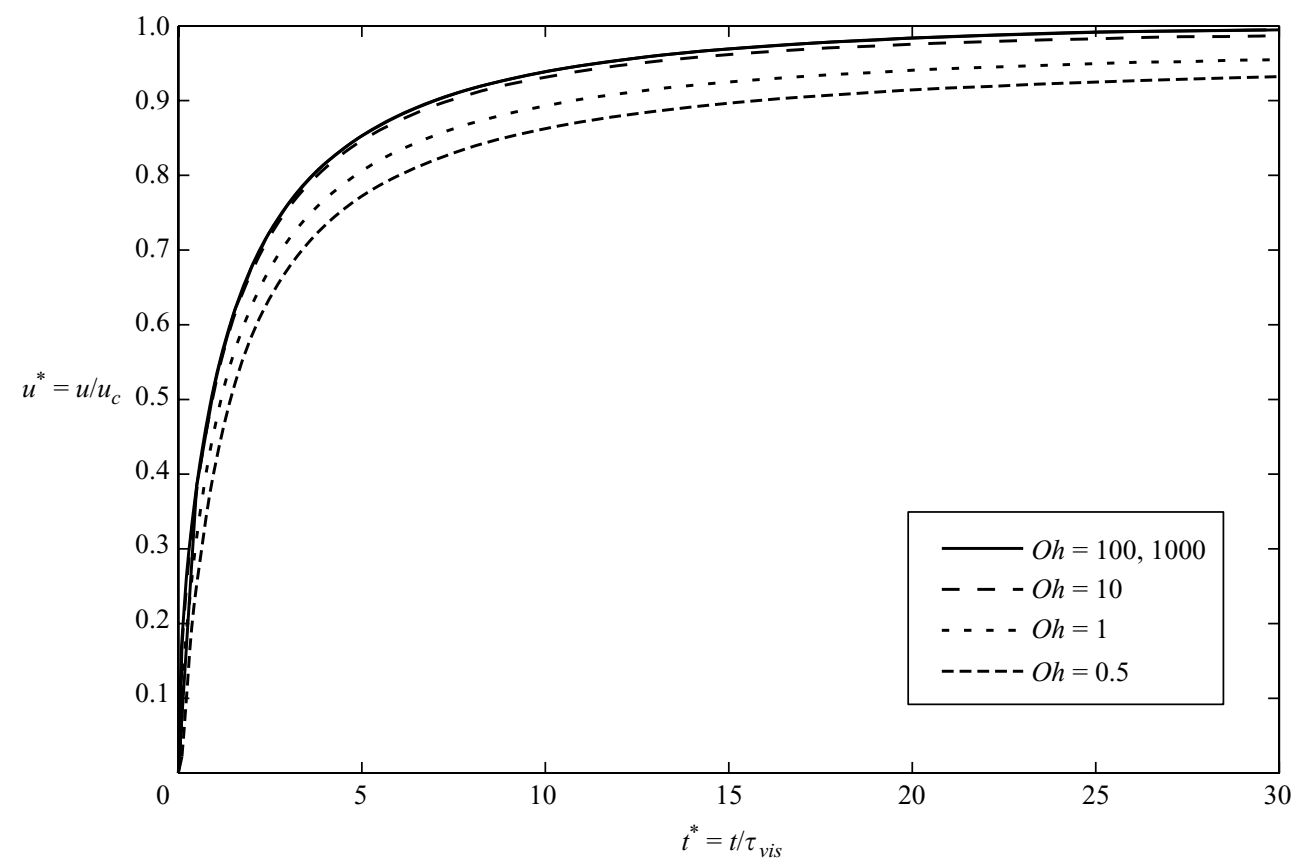

FIGURE 6. Velocity of the film edge for different Ohnesorge numbers. In the high $O$ h limit the velocity curves become indistinguishable.

preserved at all times. The dimensionless curvature is thus assumed to be

$$
\kappa^{*}=\left\{\begin{array}{cl}
-\frac{2 O h^{2}}{h_{m}^{*}(t)} & 0 \leqslant x^{*}<\frac{1}{2} h_{m}^{*}(t) / O h \\
0 & x^{*}>\frac{1}{2} h_{m}^{*}(t) / O h
\end{array}\right.
$$

The maximum film thickness is expected to be at the point of discontinuity in curvature, the centre of the semi-circular cap, where $x_{0}^{*}=(1 / 2) h_{m}^{*}(t) O h$. Plugging this expression for $\kappa^{*}$ into (2.18), integrating over an interval around $x_{0}^{*}$ and shrinking the interval to zero yields the jump condition at $x_{0}^{*}$

$$
\left[h^{*} u_{x^{*}}^{*}\right]=-\frac{1}{4} .
$$

Just to the right of $x_{0}^{*}$, we use mass conservation (2.1b) and the jump condition (2.28) to find

$$
h_{m}(t)=H\left(1+\frac{1}{4} \frac{t}{\tau_{v i s}}\right),
$$

where $H$ is the initial film thickness. The linear increase in film thickness is confirmed with the numerical results shown in figure 7. Since having a discontinuity in curvature would complicate the implementation of a numerical solution, we smooth the initial curvature gradient as discussed in the Appendix. The slope of the line is corrected to account for this smoothing by writing

$$
h_{m}=H\left(1+\frac{1}{8} H \kappa_{0} \frac{t}{\tau_{v i s}}\right),
$$

where $\kappa_{0}$ corresponds to the initial curvature at the tip. It is also important to note here the marked difference with the low $O h$ regime films, where the rim remains 


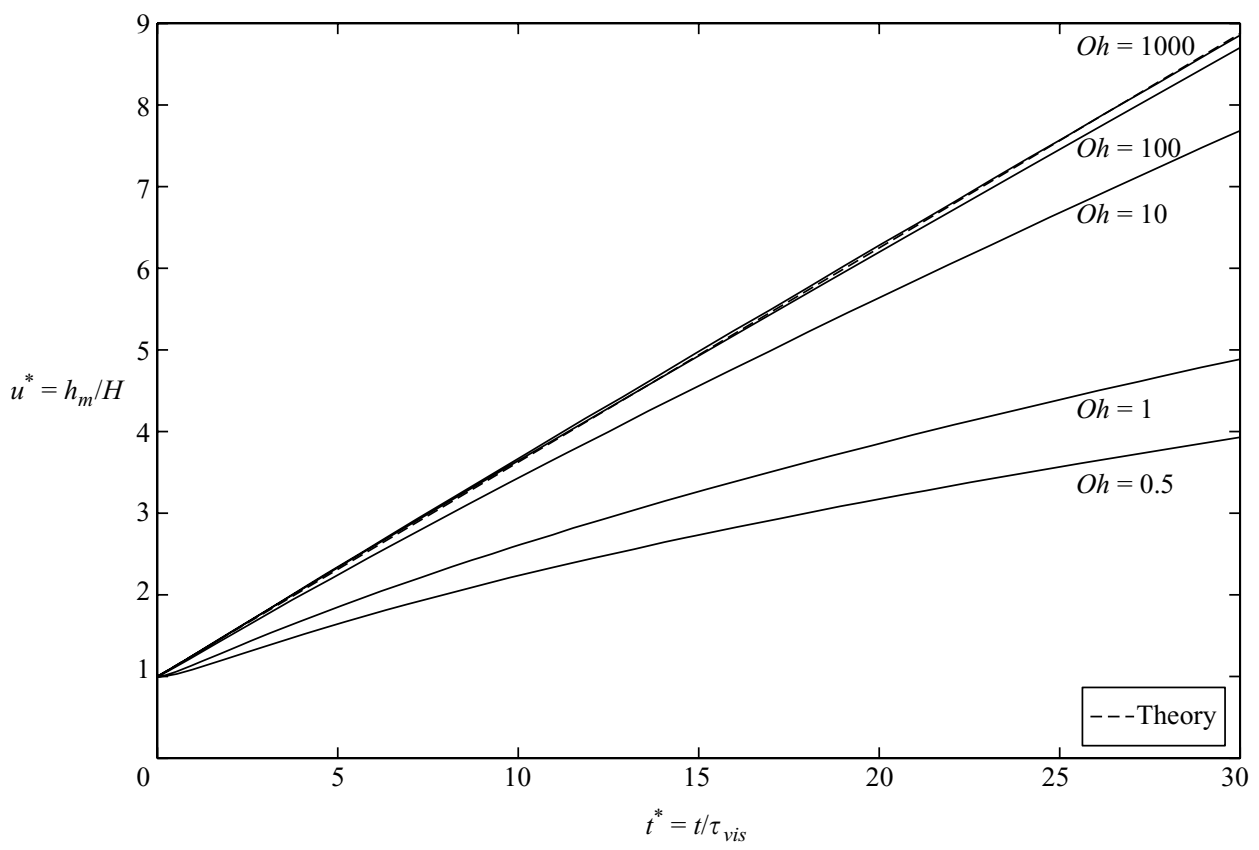

FiguRE 7. Maximum film thickness $h_{m}$ versus time for different Ohnesorge numbers. In the high $O h$ limit, $h_{m}$ grows linearly in time, confirming the theory represented by the dotted line that corresponds to (2.28). The numerical results (solid curves) at $O h=0.5,1.0,10,100$ and 1000 correspond to profiles with $\kappa_{0}=2.1$.

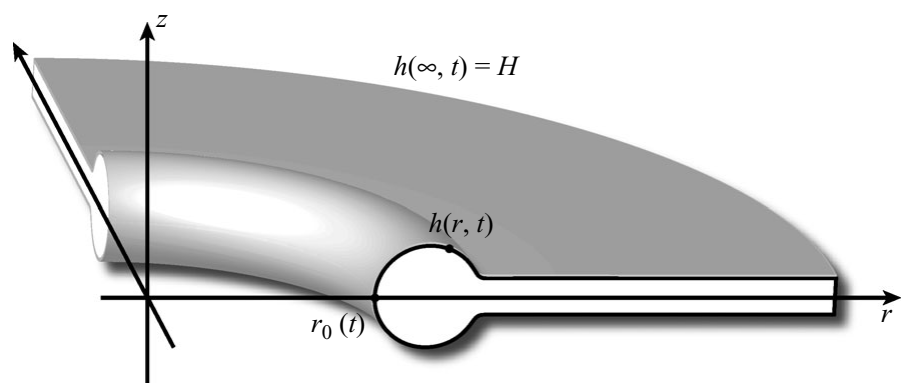

FiguRE 8. Circular sheet geometry. A circular hole expands on a flat fluid sheet owing to the radial surface tension force that acts on its toroidal edge.

circular, so mass conservation dictates that the maximum film thickness, specifically the rim radius, increases according to $\sqrt{t / \tau_{i n v}}$.

\section{Circular hole expansion}

We now turn our attention to flow in flat films initiated by nucleating a hole of radius $R_{0}$ at time $t=0$ (see figure 8). To complement the numerous experimental studies on film rupture (see for example Ranz 1950; McEntee \& Mysels 1969; Debrégeas et al. 1995; Roth et al. 2005), we develop a new theoretical model that allows us to investigate the film motion for arbitrary Ohnesorge numbers. 
Holes in thin films do not necessarily open. In an axisymmetric configuration, the azimuthal curvature contributes a component to the surface tension force that opposes hole expansion. Depending on the initial film shape and hole radius, situations may arise where the hole will contract and close, and film rupture is averted (Taylor \& Michael 1973). We shall henceforth proceed by considering configurations for which holes expand.

\subsection{Derivation of the film equations}

We consider the Navier-Stokes equations in cylindrical coordinates (Batchelor 1967)

$$
\begin{aligned}
U_{t}+U U_{r}+V U_{z} & =-P_{r} / \rho+v\left(U_{r r}+U_{z z}+U_{r} / r-U / r^{2}\right), \\
V_{t}+U V_{r}+V V_{z} & =-P_{z} / \rho+v\left(V_{r r}+V_{z z}+V_{r} / r\right),
\end{aligned}
$$

where $U$ is the radial velocity, $V$ the velocity along the axis and $P$ the fluid pressure. We neglect any azimuthal dependence on the basis of the experiments of Debrégeas et al. (1995), who confirmed that the resulting motion is purely radial via particle tracking. We also have the continuity equation

$$
(U r)_{r}+(V r)_{z}=0,
$$

together with the normal and tangential stress boundary conditions at $z=h(r, t) / 2$, respectively,

$$
\begin{array}{r}
-\frac{P}{\rho}+\left.2 v\left[U_{r} \sin ^{2} \theta-\left(V_{r}+U_{z}\right) \sin \theta \cos \theta+V_{z} \cos ^{2} \theta\right]\right|_{z=h / 2}=\frac{\gamma}{\rho} \kappa, \\
2\left(V_{z}-U_{r}\right) \sin \theta \cos \theta+\left.\left(V_{r}+U_{z}\right)\left(\cos ^{2} \theta-\sin ^{2} \theta\right)\right|_{z=h / 2}=0,
\end{array}
$$

where $\tan \theta=h_{r} / 2$ is the slope of the interface and $\kappa$ is the curvature of the boundary:

$$
\kappa(r, t)=\frac{(1 / 2) h_{r r}}{\left(1+(1 / 4) h_{r}^{2}\right)^{3 / 2}}+\frac{(1 / 2) h_{r}}{r\left(1+(1 / 4) h_{r}^{2}\right)^{1 / 2}} .
$$

Finally the kinematic boundary condition may be written as

$$
h_{t}+\left.U\right|_{z=h / 2} h_{r}=\left.V\right|_{z=h / 2} .
$$

For a slender sheet, assumed to be symmetric about $z=0$, we use a Taylor expansion about $z=0$, to write

$$
\begin{aligned}
& U(r, z, t)=u(r, t)+u_{2}(r, t) z^{2}+\cdots, \\
& V(r, z, t)=v_{1}(r, t) z+v_{3}(r, t) z^{3}+\cdots, \\
& P(r, z, t)=p(r, t)+p_{2}(r, t) z^{2}+\cdots .
\end{aligned}
$$

This approach was successfully used in the past to study jet breakup (Eggers \& Dupont 1994; Eggers \& Brenner 2000). Matching powers of $z$ in (3.3) we find that to the lowest order in $z$

$$
\begin{aligned}
& v_{1}=-\left(u_{r}+\frac{u}{r}\right), \\
& v_{3}=-\frac{1}{3}\left(u_{2 r}+\frac{u_{2}}{r}\right) .
\end{aligned}
$$


Similarly, (3.1) and (3.2) yield

$$
\begin{gathered}
u_{t}+u u_{r}=-p_{r} / \rho+v\left(u_{r r}+u_{r} / r-u / r^{2}+2 u_{2}\right), \\
v_{1 t}+u v_{1 r}+v_{1}^{2}=-2 p_{2} / \rho+v\left(v_{1 r r}+v_{1 r} / r+6 v_{3}\right) .
\end{gathered}
$$

In the long wavelength limit, $h \gg h_{r}$ we find that to leading order in $h$ the boundary and kinematic conditions, (3.4a), (3.4b) and (3.6) become, respectively,

$$
\begin{aligned}
-\frac{p}{\rho} & =\frac{\gamma}{\rho} \kappa-2 v v_{1}, \\
2 u_{2} & =-2 \frac{h_{r}}{h}\left(v_{1}-u_{r}\right)-v_{1 r}, \\
h_{t}+u h_{r} & =v_{1} h .
\end{aligned}
$$

We eliminate $v_{1}$ in (3.14) using (3.8) to find

$$
h_{t}+\frac{1}{r}(u r h)_{r}=0 .
$$

Similarly, elimination of $u_{2}$ and $p$ from (3.10) using (3.12), (3.13) and (3.8) yields

$$
u_{t}+u u_{r}=\frac{4 v}{h}\left(\left(\frac{h}{r}(u r)_{r}\right)_{r}-\frac{u h_{r}}{2 r}\right)+\frac{\gamma}{\rho} \kappa_{r} .
$$

Equations (3.15) and (3.16) constitute a system of lubrication equations that describe the retraction of a circular sheet. However this long-wavelength approximation is not valid everywhere and the equations become singular as we approach the film tip. Similar difficulties arise in various applications of the lubrication approximation; nevertheless, such models perform surprisingly well. For instance, in Eggers \& Dupont (1994), the lubrication model yields excellent agreement with experimental observations of jet breakup and pendant drop formation.

\subsection{Early stages of expansion}

We proceed by performing an early-time analysis of the governing equations (3.15) and (3.16) in the high $\mathrm{Oh}$ limit, by following the ideas developed in $\S 2.2$ for planar sheets. Introducing the scalings

$$
r=O h H r^{*}, h=H h^{*}, u=u_{c} u^{*}, R_{0}=O h H R_{0}^{*} \text { and } t=\tau_{v i s} t^{*},
$$

we write (3.16) in non-dimensional form as

$$
u_{t^{*}}^{*}+u^{*} u_{r^{*}}^{*}=\frac{4}{h^{*}}\left[\left(\frac{h^{*}}{r^{*}}\left(u^{*} r^{*}\right)_{r^{*}}\right)_{r^{*}}-\frac{u^{*} h_{r^{*}}}{2 r^{*}}\right]+\frac{1}{2} O h^{-2} \kappa_{r^{*}}^{*} .
$$

We assume that initially the rim corresponds to the inner portion of a torus and matches onto a flat sheet. The film curvature thus becomes

$$
\kappa^{*}=\left\{\begin{array}{cl}
2 O h^{2}\left(-1+\frac{r_{s}^{*}-r}{r}\right) & R_{0}^{*} \leqslant r^{*}<r_{s}^{*} \\
0 & r^{*}>r_{s}^{*}
\end{array}\right.
$$

where $r_{s}^{*}=R_{0}^{*}+(1 / 2) O h^{-1}$ is the point where the rim and the flat sheet meet. Note here the second term in the expression for the curvature that arises from the axisymmetry of the problem. Dropping the stars for convenience, we find that in the same limit considered for early-stage planar sheet retraction (i.e. convective terms negligible, and $h$ constant for $r>r_{s}$ ) the same jump condition arises, namely,

$$
\left[u_{r}\right]=-\frac{1}{4} .
$$


To the right of the jump, $h$ variations are neglected, so we consider

$$
(u r)_{t}=4\left[(u r)_{r r}-\frac{(u r)_{r}}{r}\right],
$$

which can be solved by separation of variables to yield

$$
u=A \mathrm{e}^{\lambda^{2} t} K_{1}\left(\frac{1}{2} \lambda r\right),
$$

where $A$ and $\lambda$ are constants to be determined and $K_{1}$ is the modified Bessel function of the second kind of order 1 . Note that the modified Bessel function of the first kind $I_{1}$ diverges as $r \rightarrow \infty$ and is thus dropped since $u \rightarrow 0$ at infinity. Denoting the radius of the punctured hole and its time derivative by $r_{0}$ and $\dot{r}_{0}$, respectively, we take $r_{s} \approx r_{0}(t)$ to obtain

$$
\dot{r_{0}}=A \mathrm{e}^{\lambda^{2} t} K_{1}\left(\frac{1}{2} \lambda r_{0}\right)
$$

upon application of the boundary condition $u\left(r_{0}, t\right)=\dot{r_{0}}(t)$. To proceed further, we note that the argument of $K_{1}, \lambda r_{0} / 2$, is small during early stages of retraction. By retaining only the first-order term in the small argument expansion of $K_{1}$, we find

$$
\dot{r}_{0}=\frac{2 A \mathrm{e}^{\lambda^{2} t}}{\lambda r_{0}},
$$

which gives $r_{0}(t)=R_{0} \mathrm{e}^{\lambda^{2} t / 2}$. This allows us to write the sheet speed to the right of the cap as

$$
u=\frac{\lambda^{3} R_{0}^{2}}{4} \mathrm{e}^{\lambda^{2} t} K_{1}\left(\frac{1}{2} \lambda r\right) .
$$

Just to the right of the jump, we calculate the velocity gradient at $r=r_{s} \approx r_{0}$ and expand the derivative of $K_{1}$ to obtain

$$
u_{r}\left(r_{s}^{+}\right)=-\lambda^{2} / 2
$$

to leading order in $r_{0}$. Thus far, $\lambda$ remains unknown and needs to be determined by the jump condition. Unlike the planar case, there are non-zero velocity gradients within the cap when $r_{0}$ is sufficiently small. Solving for the flow within the cap cannot be done analytically, but we note that the term $h_{r} / h$ dominates the other terms in (3.18), which thus assumes the form

$$
\frac{h_{r}}{h}\left[\frac{(u r)_{r}}{r}-\frac{u}{2 r}\right]=0 .
$$

By requiring the continuity of $u(r, t)$ at $r=r_{0}$, this equation may be solved for $u$ to yield

$$
u=\frac{\lambda^{2} R_{0}^{3 / 2}}{2 r^{1 / 2}} .
$$

The velocity gradient at $r=r_{s}^{-}$is thus

$$
u_{r}\left(r_{s}^{-}\right)=-\lambda^{2} / 4 .
$$

Using (3.26) and (3.29) in (3.20) we find that $\lambda=1$. We therefore conclude that the hole grows exponentially during the early stages of retraction

$$
r_{0}(t)=R_{0} \exp \left(\frac{t}{2 \tau_{v i s}}\right),
$$


as expressed in dimensional variables. Hence the exponential hole growth reported by Debrégeas et al. (1995) need not be attributed to viscoelastic effects; rather, it is a generic feature of circular hole retraction on a viscous sheet. We note that our approach, based entirely on the lubrication equations, complements the energy argument of Debrégeas et al. (1995).

\subsection{Simulation results}

The governing equations are solved numerically as described in the Appendix. Even though we explored a wide range of $O h$, particular attention was given to the high Oh regime, in order to make comparisons with recently reported experimental work (e.g. Debrégeas et al. 1995; Dalnoki-Veress et al. 1999; Roth et al. 2005). The regimes identified in the work of Brenner \& Gueyffier (1999) are also present in the retraction of circular sheets (figure 2). While the differences between the two geometries are not significant at low $\mathrm{Oh}$, there are striking differences in the retraction dynamics in the high $\mathrm{Oh}$ regime. For the sake of clarity of presentation, we devote $\S \S 3.3 .1,3.3 .2$ and 3.3.3 to discussions of the high $(O h \gg 10)$, moderate $(0.01<O h<10)$ and low $(O h<0.01)$ Ohnesorge number regimes, respectively.

\subsubsection{High Oh simulations}

Experiments in the high $\mathrm{Oh}$ limit have been limited to fluids with long chain polymers, specifically PDMS in Debrégeas et al. (1995) and molten polystyrene in Dalnoki-Veress et al. (1999) and Roth et al. (2005). We proceed by demonstrating that the essential features of retraction reported are captured by a Newtonian fluid description.

Figure 9 illustrates the evolution of typical film profiles and midplane velocities at various $O h$. As $O h$ increases beyond 100, the rim diffuses towards the bulk of the film, thus making the film appear to be of uniform thickness. We note that even at high $O h$, the film is slightly thicker near the rim and very gradually thins further away from the tip. It is also important to note that the region of influence of the tip motion, which grows in time as more fluid is set into motion, is directly proportional to $\mathrm{Oh}$.

Just as the velocity curves for a planar sheet asymptote to a single curve in the high $O h$ limit (see figure 6), something similar can be said for the circular sheet. However, the initial size of the nucleated hole must now be considered. In particular, having written the momentum equations in non-dimensional form using (3.17) and by assuming that the film profile near the tip is preserved, we can replace the derivative of the film curvature with the approximate expression

$$
\kappa_{r^{*}}^{*} \approx 2 O h^{2} \delta\left(r-r_{0}^{*}\right),
$$

provided that the azimuthal curvature term in (3.5) is much smaller than the curvature of the film profile, i.e. $H / R_{0} \ll 1$. Doing so leaves us with a set of dimensionless equations that are independent of $O h$. Therefore, different simulations will yield almost identical results provided that $r_{0}^{*}$, the dimensionless initial hole radius, is the same. In other words, curves that have the same ratio $H O h / R_{0}$ will yield virtually indistinguishable self-similar velocity curves, as confirmed by our simulations. For example, tip speeds of a simulation with $\mathrm{Oh}=10^{4}$ and $R_{0}=50 \mathrm{H}$ are indistinguishable from these with $\mathrm{Oh}=10^{3}$ and $R_{0}=5 \mathrm{H}$.

Figure 10 indicates the dependence on $O h$ of the approach of the sheet tip to the Taylor-Culick speed. High $\mathrm{Oh}$ films require substantially more time than low $\mathrm{Oh}$ films to approach $u_{c}$. Viscous forces delay the acceleration process, because a larger 
(a)
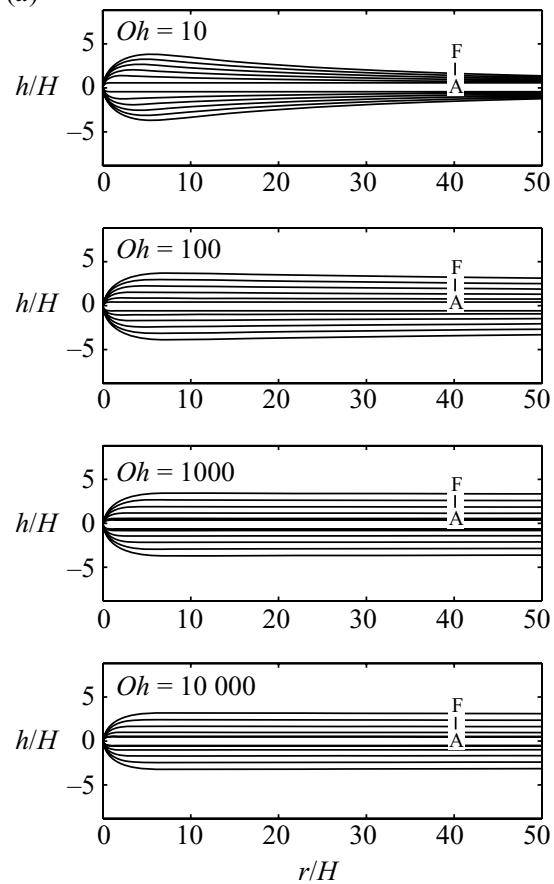

(b)
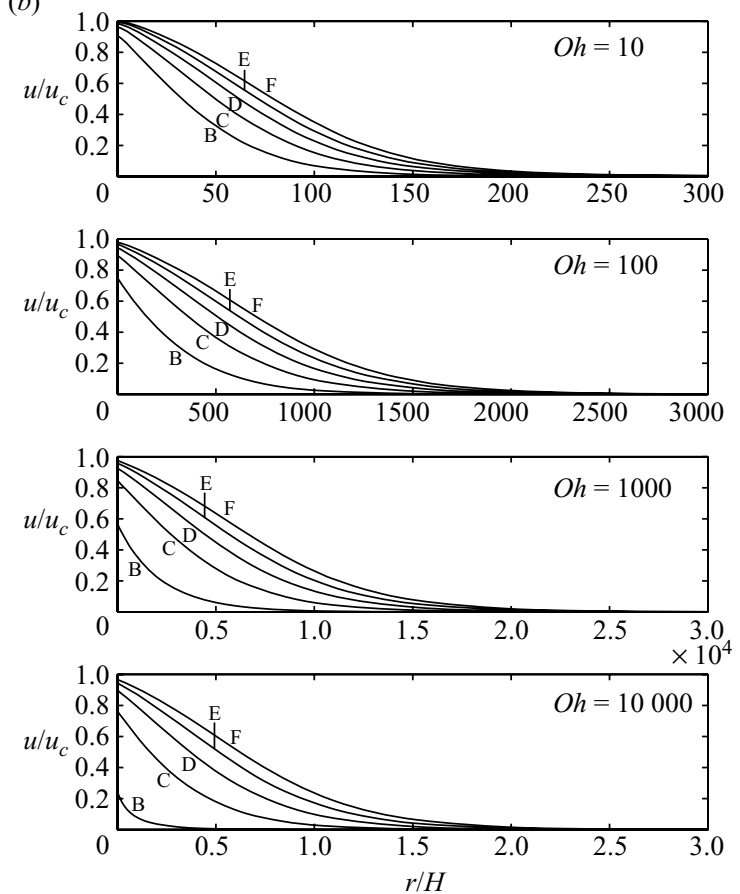

FIGURE 9. Evolution of the film boundaries (panel $a$ ) and midplane speeds (panel $b$ ) for high $O h$ and initial hole size $R_{0}=50 \mathrm{H}$ in time increments of $\delta t=10 \tau_{v i s}$. Curves A to F correspond to times $t^{*}=t / \tau_{v i s}=0,10,20,30,40$ and 50 , respectively. Distances are scaled by the film thickness $H$. As $O h$ is progressively increased from 10 to $10^{4}$, the rim becomes less pronounced and diffuses towards the bulk. Concurrently, the region of influence of the disturbance caused by the tip motion extends further into the film and its lateral extent is proportional to $O h$.

amount of fluid needs to be accelerated by surface tension forces concentrated near the film edge.

In figure 11 we confirm the exponential retraction rate observed in the experiments (see Debrégeas et al. 1995; Dalnoki-Veress et al. 1999; Roth et al. 2005) during the early stages of retraction. We note that the higher the $\mathrm{Oh}$, the longer the hole grows according to the exponential law. By comparison with figure 10, we infer from our simulations that the deviation from the exponential behaviour occurs quite early in the retraction process, typically when the rim speed is of the order of $0.2 u_{c}$.

In order to assess how $\mathrm{Oh}$ affects the acceleration phase, we plot in figure 12 the time required for the tip to attain $30 \%, 60 \%$ and $90 \%$ of the Taylor-Culick speed for different $O h$. Each simulation was initialized with a hole radius of $R_{0}=50 H$. As can be inferred from the theoretically predicted exponential law (1.3), the times $t_{1,2}$ needed for the tip to attain the speed of a certain fraction of $u_{c}$ that correspond to $O h_{1,2}$ satisfy the relation

$$
\frac{t_{1}}{O h_{1}}-\frac{t_{2}}{O h_{2}}=2 \log \left(\frac{O h_{1}}{O h_{2}}\right)
$$

provided $O h_{1,2} \geqslant 100$ and the punctured hole is not large. At lower $O h$, there is a clear deviation from this relation, which indicates that the rim acceleration is different from that predicted in the viscous limit. 


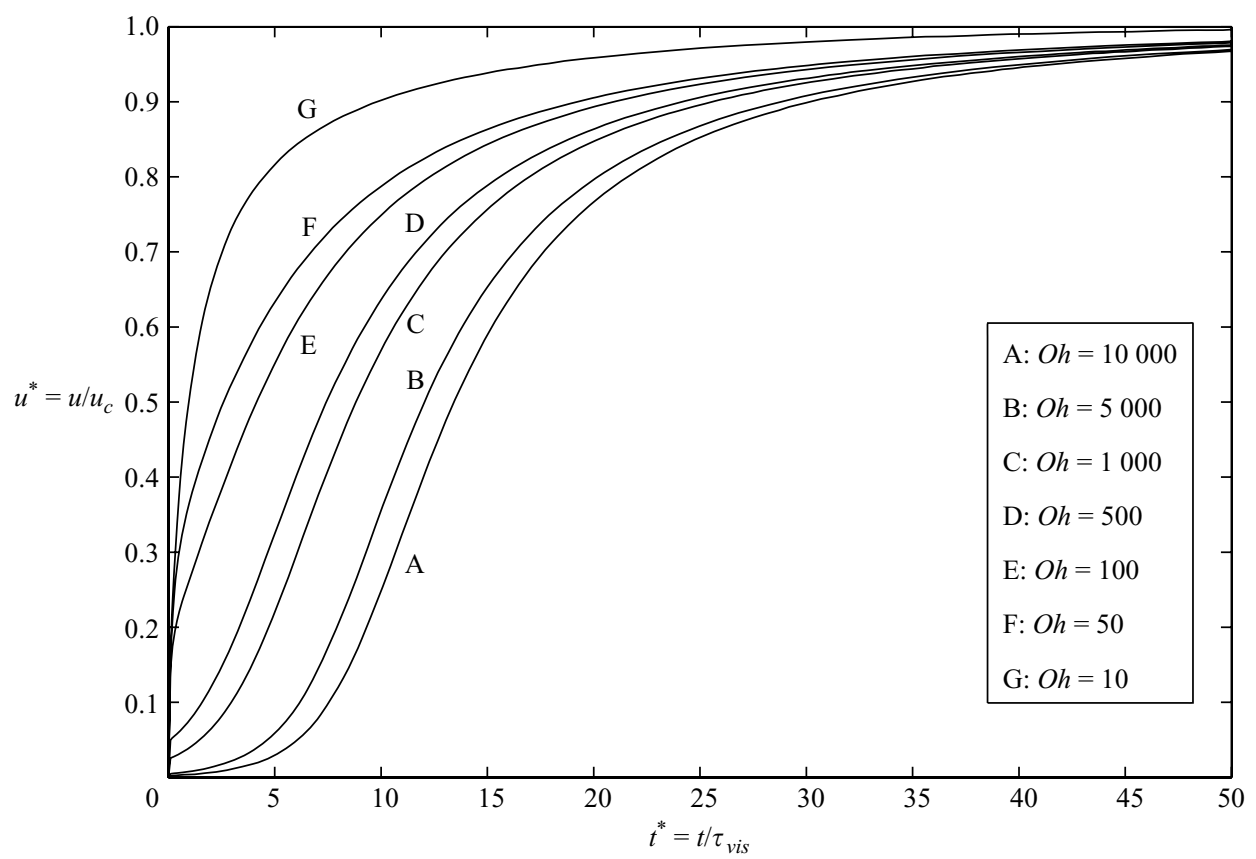

FiguRE 10. Speed at the film edge as a function of time for different values of $O h$, with initial hole radius of $R_{0}=50 \mathrm{H}$. The higher the $\mathrm{Oh}$ the slower the approach towards the Taylor-Culick speed $u_{c}$.

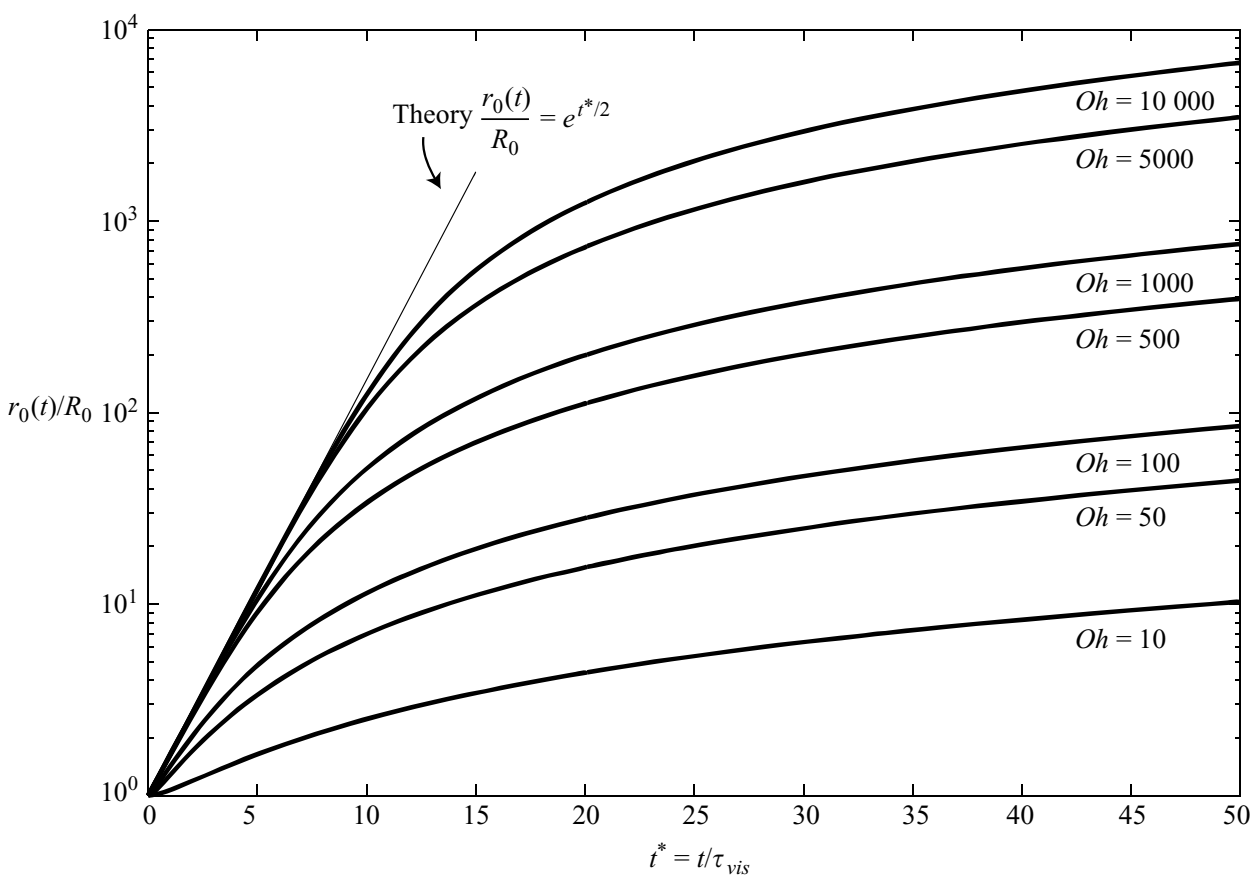

Figure 11. Hole growth versus time for different $O h$, for an initial hole size $R_{0}=50 H$. The higher the $\mathrm{Oh}$, the longer the hole grows according to the exponential law (1.3) as deduced by Debrégeas et al. (1995). 


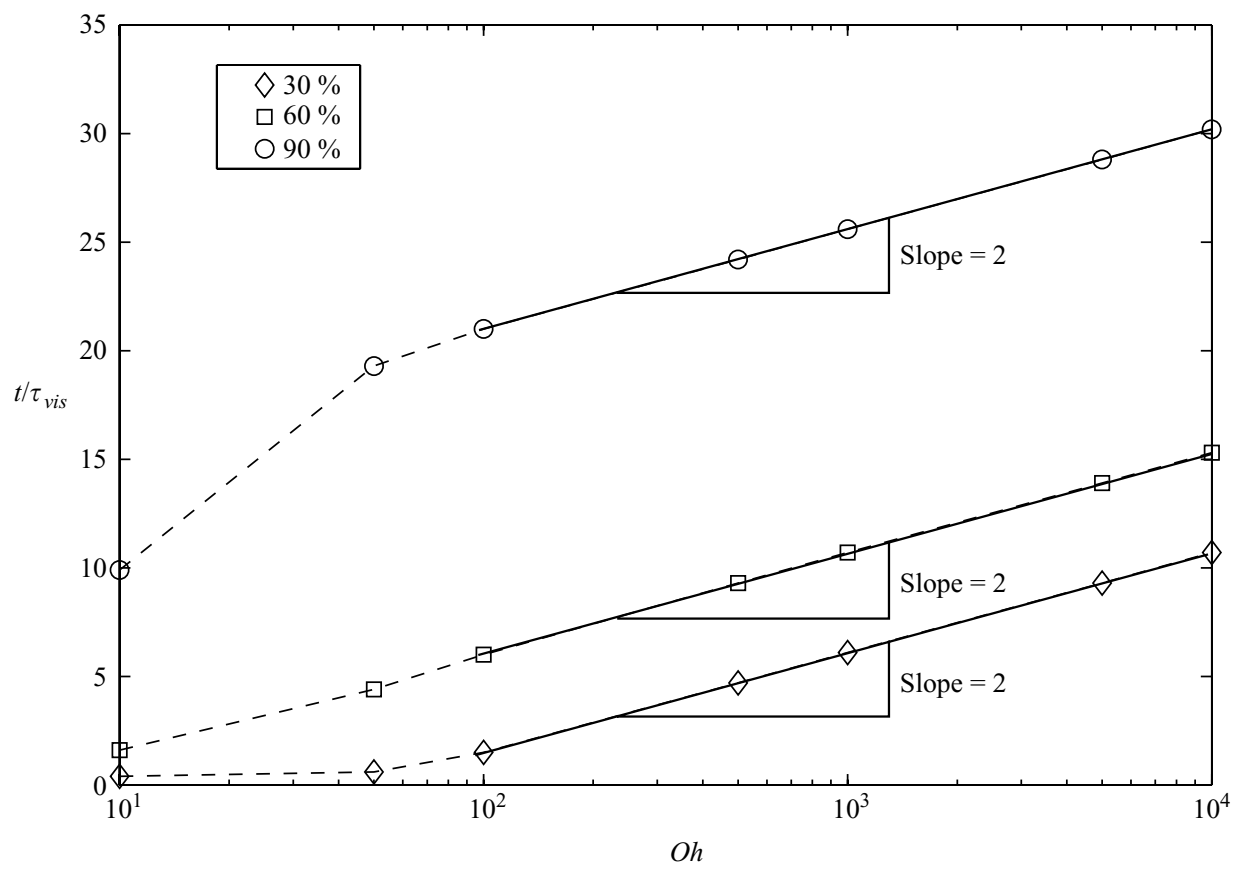

FIGURE 12. Time to reach $30 \%, 60 \%$ and $90 \%$ of the Taylor-Culick speed versus $\mathrm{Oh}$ for simulations with $R_{0}=50 \mathrm{H}$. For $O h \geqslant 100$, there is logarithmic dependence on the Ohnesorge number, confirming the theoretically predicted retraction rate (1.3).
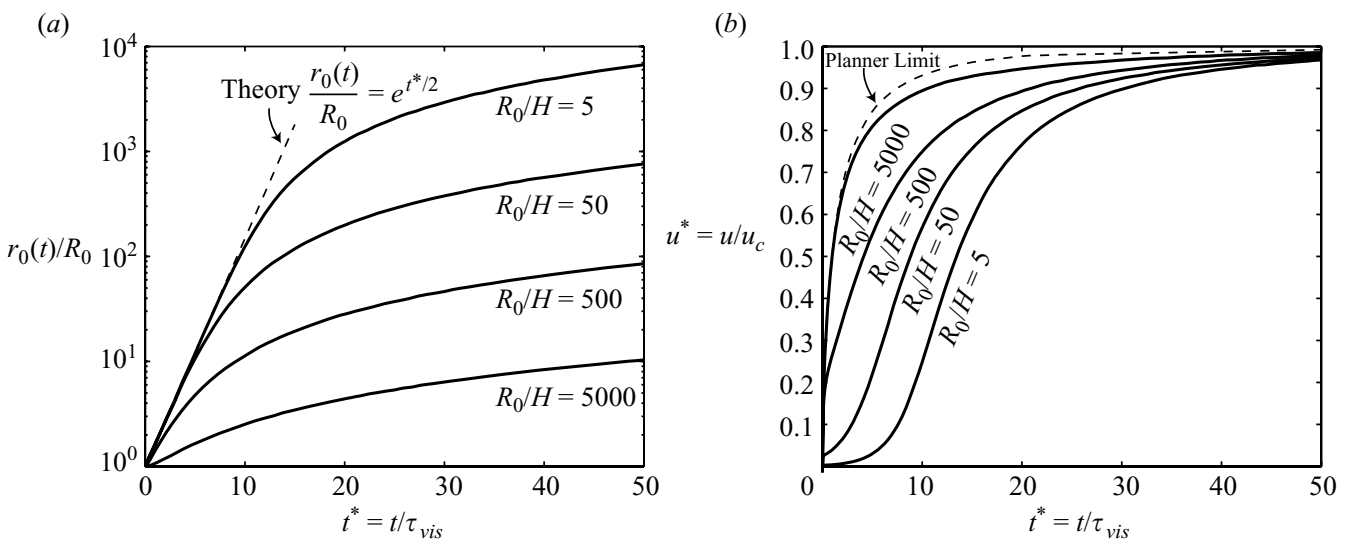

FIgURE 13. Variation of initial hole size for $O h=1000:(a)$ hole radius versus time and $(b)$ tip speed versus time. For small initial hole radii, the agreement with the theoretically predicted exponential rate lasts for longer times. For larger initial radii, the retraction speed rapidly approaches the planar limit considered in $\S 2$.

We proceed by looking at the effect of the initial hole size on the retraction dynamics. Figure 13(a) shows the evolution of the hole radius for a number of different initial radii, when $O h=1000$. The calculations indicate that the smaller the initial hole size, the longer the hole radius grows exponentially according to (1.3). As expected, the circular geometry is thus influencing the dynamics for longer when the initial hole radius is smaller: when the hole radius is large, the film motion rapidly 


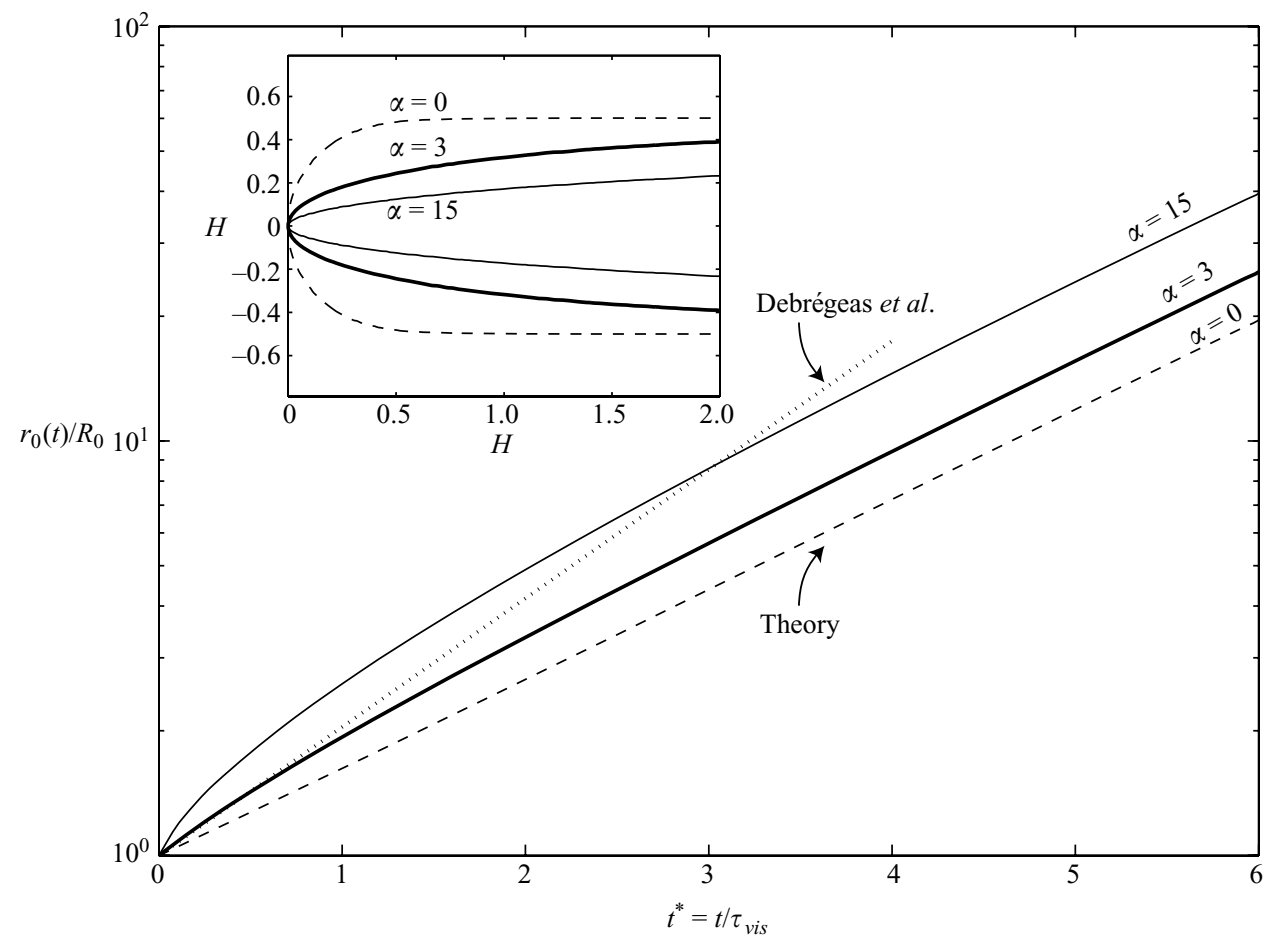

FIGURE 14. Effect of the initial film profile (as specified by $\alpha$ ) on sheet retraction. Hole radius versus time for $O h=8 \times 10^{3}$ and $R_{0}=40 \mathrm{H}$. The inset shows the corresponding initial film profiles in the vicinity of the tip. A more pointed initial film profile retracts faster until its tip relaxes to a semi-circular cap; thereafter, the film retracts at the theoretically predicted rate shown by the dashed curve, corresponding to (3.30). The dotted line indicates the experimentally observed retraction rates reported by Debrégeas et al. (1995).

approaches the planar limit considered in $\S 2$ (see figure $13 b$ ). Variations in the initial hole radius affect the low $\mathrm{Oh}$ films to a lesser extent, mainly due to the shorter time scales involved in the approach to $u_{c}$.

The discrepancy between the time scale of hole growth deduced by Debrégeas et al. (1995), $1.4 \tau$ (see (1.2)), and that predicted by theory, $2 \tau$ (see (1.3)), prompts us to look at the effect of the initial film profile on the initial stages of retraction. The initial film profile near the tip need not be semi-circular. We adopt the film profile of (A 8) discussed in the Appendix, whose sharpness can be conveniently modified via a single parameter, $\alpha>0$. As $\alpha \rightarrow 0$, the tip approaches a circular cap; the curvature at the tip increases with $\alpha$ according to (A9). In figure 14, we show the effect on the retraction rate as the initial tip profile becomes progressively more pointed. Sheets with more pointed tips initially retract faster owing to the enhanced surface tension force. Eventually as the rim acquires a more circular form, the retraction slows down to the rate predicted by theory. Whether the pointed initial profile is the source of the anomalously large retraction rate observed by Debrégeas et al. (1995) cannot be assessed due to uncertainty in the film shape and the limited duration over which their data was collected.

Roth et al. (2005) similarly observed that the rate of retraction is faster at the onset of retraction; fitting of the experimental data was achieved by assuming an empirically deduced time-dependent viscosity. However, our calculation yields a behaviour that 


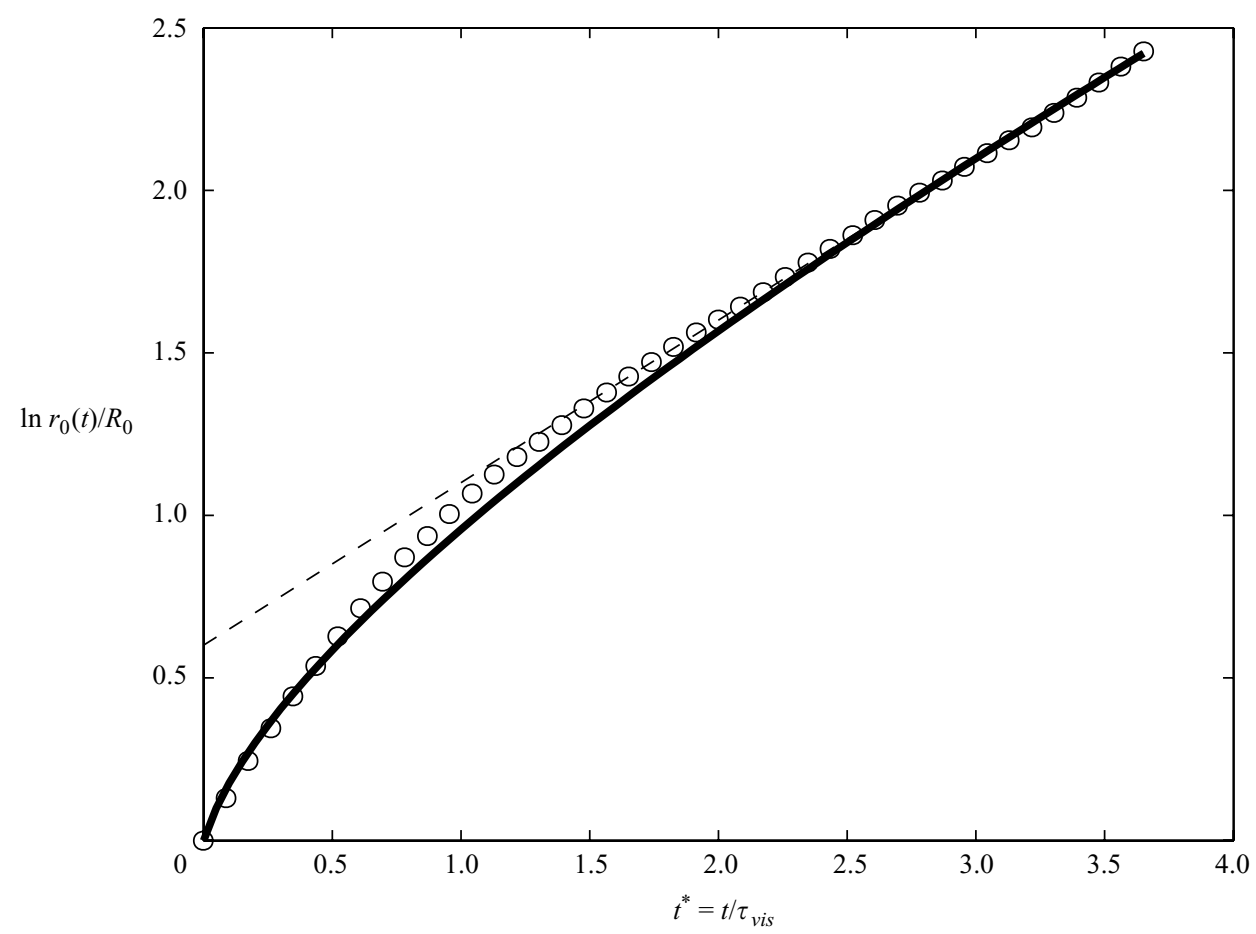

FIGURE 15. Hole radius versus time. The experimental data of Roth et al. (2005) is presented as circles, and our simulations for $O h=1000$ and $\alpha=32$ by a solid line. After an initial transient of order $\tau_{v i s}=\mu H / 2 \gamma$, the retraction rate decreases to that predicted by (3.30), corresponding to the dashed line.

is similar to their experimental observations, suggesting that the initial retraction rate they observe might be reasonably attributed to the initial puncture shape. This is demonstrated in figure 15, where we show the experimental results of Roth et al. (2005) with the results of our simulations. As a caveat, we note that the Ohnesorge number of the experiments was of the order of $10^{12}$, a value not attainable in our simulations. Nevertheless, using a pointed initial profile for $O h=1000$ and $\alpha=32$ we confirm that the behaviour observed in the experiments of Roth et al. (2005) can also arise for Newtonian films.

\subsubsection{Moderate Oh simulations}

Moderate $O h$ films $(0.01<O h<10)$ arise in most configurations of practical importance, for example the bursting of soap films. In figure 16 we show the evolution of the tip velocity for different $O \mathrm{~h}$, when the initial hole radius is $R_{0}=50 \mathrm{H}$. Not much can typically be said about the acceleration phase of the film edge because it happens extremely rapidly, on a timescale of the order of microseconds. For comparison, we included the calculation for a high $O h$ film $(O h=100)$ to illustrate that the transition to $u_{c}$ happens on a much longer time scale at moderate $O h$. It is also worth noting that in the $O h=0.04$ case, there is temporarily a slight reduction in the acceleration of the tip, because of the capillary waves generated ahead of the rim. These waves transfer momentum upstream of the advancing rim and the associated wave drag presumably slows down the rim.

In figure 17 we show the evolution of typical film profiles and radial midplane velocities. At low $\mathrm{Oh}$, the motion is more localized to the vicinity of the edge. The 


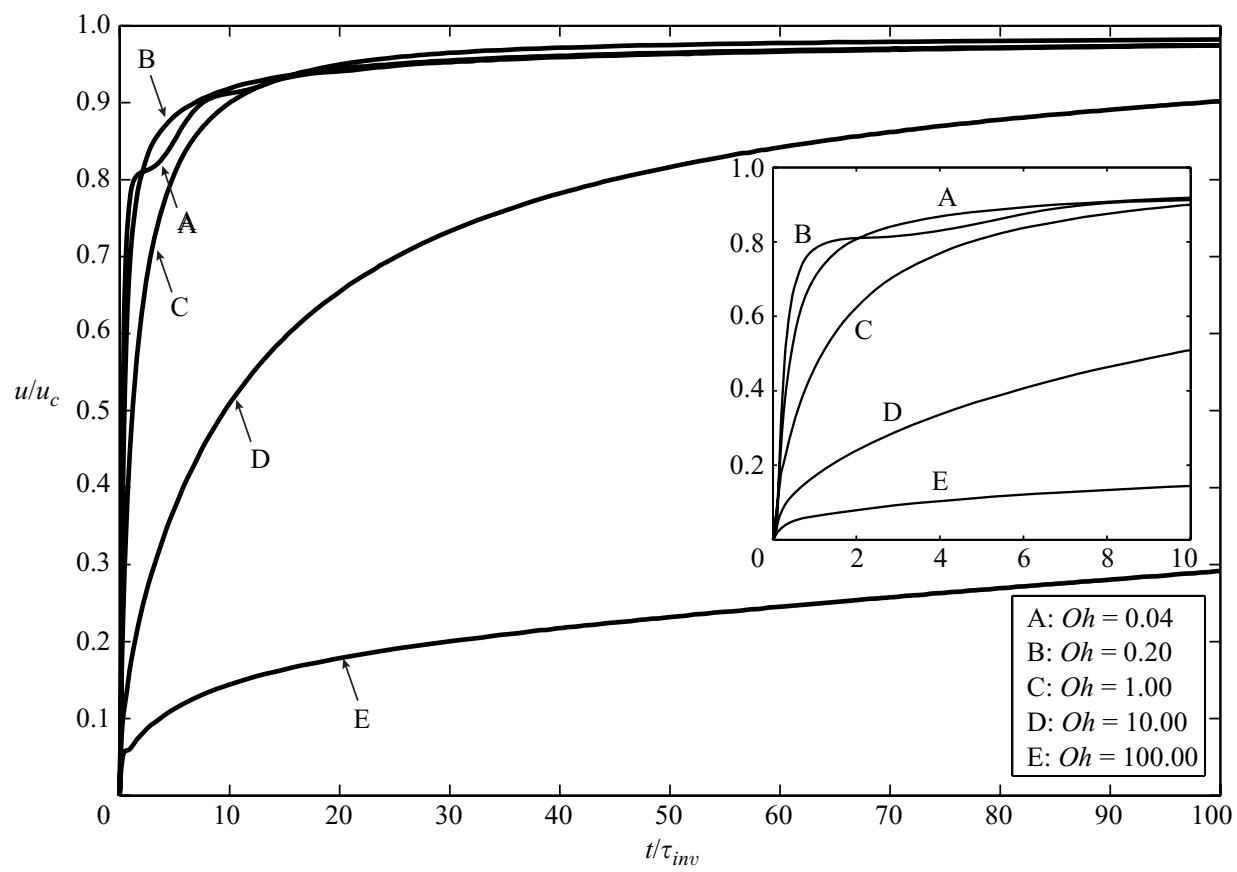

FigURE 16. Speed of the film tip versus time since rupture for moderate $O h$. Note the relatively slow retraction of the $O h=100$ film. (Inset) Early stages of retraction. When $O h=0.04$, there is a brief reduction in the rate at which the film approaches $u_{c}$, due to the production of capillary waves ahead of the rim.

surface tension forces impart acceleration to the rim, which in turn collides with the quiescent fluid in the bulk of the film. The inertia of the fluid ahead of the advancing edge is thus responsible for rim growth. Film retraction at $O h<0.1$ is accompanied by capillary waves that precede the rim. We note that similar behaviour was reported in the inertial dewetting of thin films by Buguin, Vovelle \& Brochard-Wyart (1999). The work of Song \& Tryggvason (1999) provided some physical insights regarding rim and capillary wave formation on planar sheets. In particular, via two-dimensional simulations, they argued that the curvature variations near the tip generate local vorticity that initiates the retraction of the edge. For high $O h$ films, this vorticity diffuses through the bulk of the film so that no rim forms.

While the study of vorticity in our one-dimensional model is not possible, we can gain some insights into the flow by constructing an approximation to the velocity field using the expansions from $\S 3.1$. Using the velocity $u$ and film thickness $h$ obtained from our simulations, we plot the velocity field $(U, V)$ along the $(r, z)$ directions in polar coordinates and the pressure along the midplane of the film using the set of equations (3.7). Even though the computation of $u$ and $h$ is second-order accurate in $\delta r$ and the computations for $U$ and $V$ involve the evaluation of higher derivatives of $u$, it is possible to capture the essential features of the flow. Figure 18 shows the velocity field in the film together with the pressure along the midplane for films in the three regimes of interest. In the high $\mathrm{Oh}$ regime (Figure 18a), the pressure field attains its maximum near the film tip and monotonically decreases towards the bulk. This pressure gradient essentially generates the flow that drives the retraction. As the $\mathrm{Oh}$ is decreased (Figure 18b), we see that the pressure is nearly constant in the rim and 
(a)
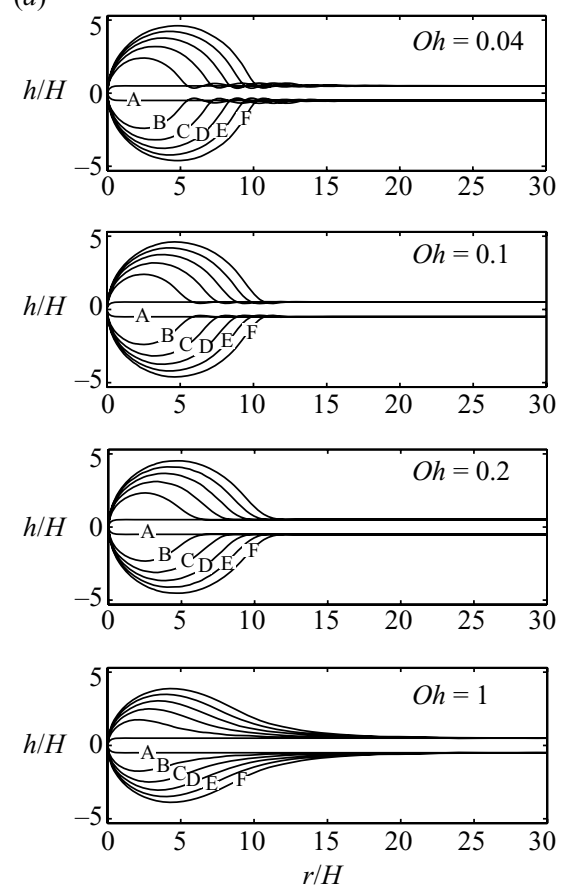

(b)
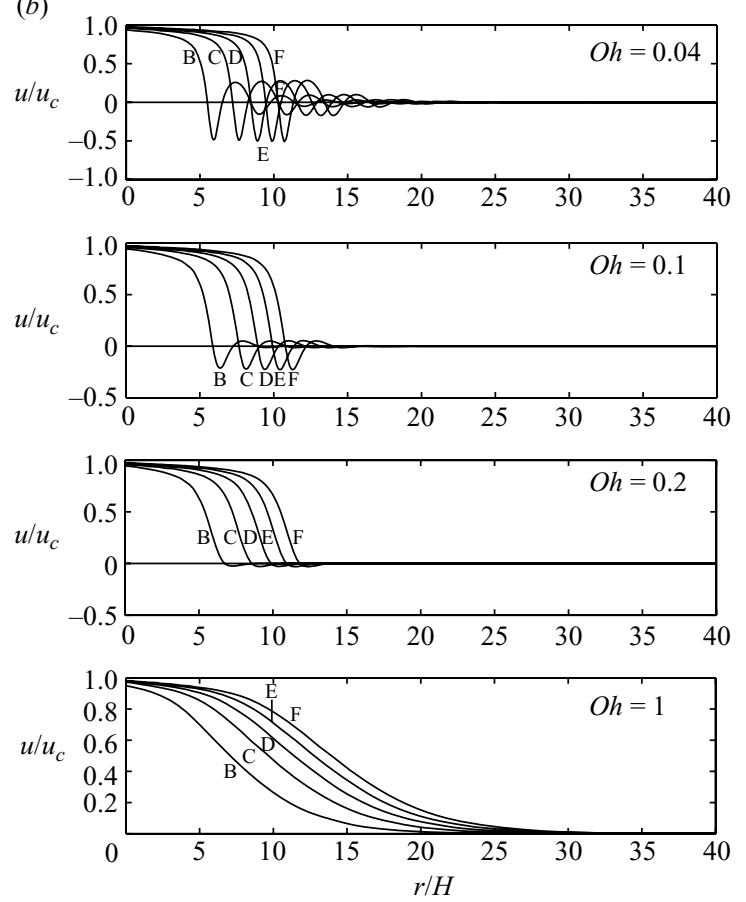

FIGURE 17. Evolution of the film boundaries and midplane velocities for moderate $O h$ in time increments of $\delta t=20 \tau_{i n v}$. Curves A to $\mathrm{F}$ correspond to times $t^{*}=t / \tau_{i n v}=0,20,40,60,80$ and 100 , respectively. The initial hole radius is $R_{0}=50 \mathrm{H}$. Note the generation of the capillary waves that are most pronounced for $\mathrm{Oh}=0.04$ and the associated oscillations in the midplane velocities. As $O h$ is increased, the capillary waves diminish and the rim begins to diffuse towards the bulk of the film.

then drops at the junction of the rim and the sheet. From the velocity field in the lab frame we notice a weak backflow towards the rim that necessarily contributes to rim growth. For the low $\mathrm{Oh}$ film (Figure 18c) at each successive neck there are pressure drops and we see circulating flow, confirming the local generation of vorticity.

\subsubsection{Low Oh simulations}

The situation is markedly different for $O h<0.04$. A simulation was run for $O h=0.01$, frames of which are shown in figure 19. As expected, the capillary waves generated are of higher amplitude than the $O h=0.04$ case. When these waves grow sufficiently in amplitude, they begin to interact with the growing rim. As time progresses, this interaction becomes more violent, causing the thickness of the neck near the rim to approach zero. At $t=100 \tau_{i n v}$, when the simulation was terminated, the film was on the verge of pinch-off: a sharp curvature arose that was barely resolved with our grid spacing.

The inset of figure 19 shows the evolution of the tip speed, which oscillates with an amplitude that increases over time, as the rim interacts with the waves it generates. This can be compared with the $O h=0.04$ film in figure 16, where the influence of waves is only apparent during a short initial interval, and the tip speed increases monotonically towards $u_{c}$. An indication exists in the work of Song \& Tryggvason (1999) that this wave-rim interaction can occur for $O h=O(0.01)$; however, their twodimensional simulations were not carried out long enough to see whether it becomes 

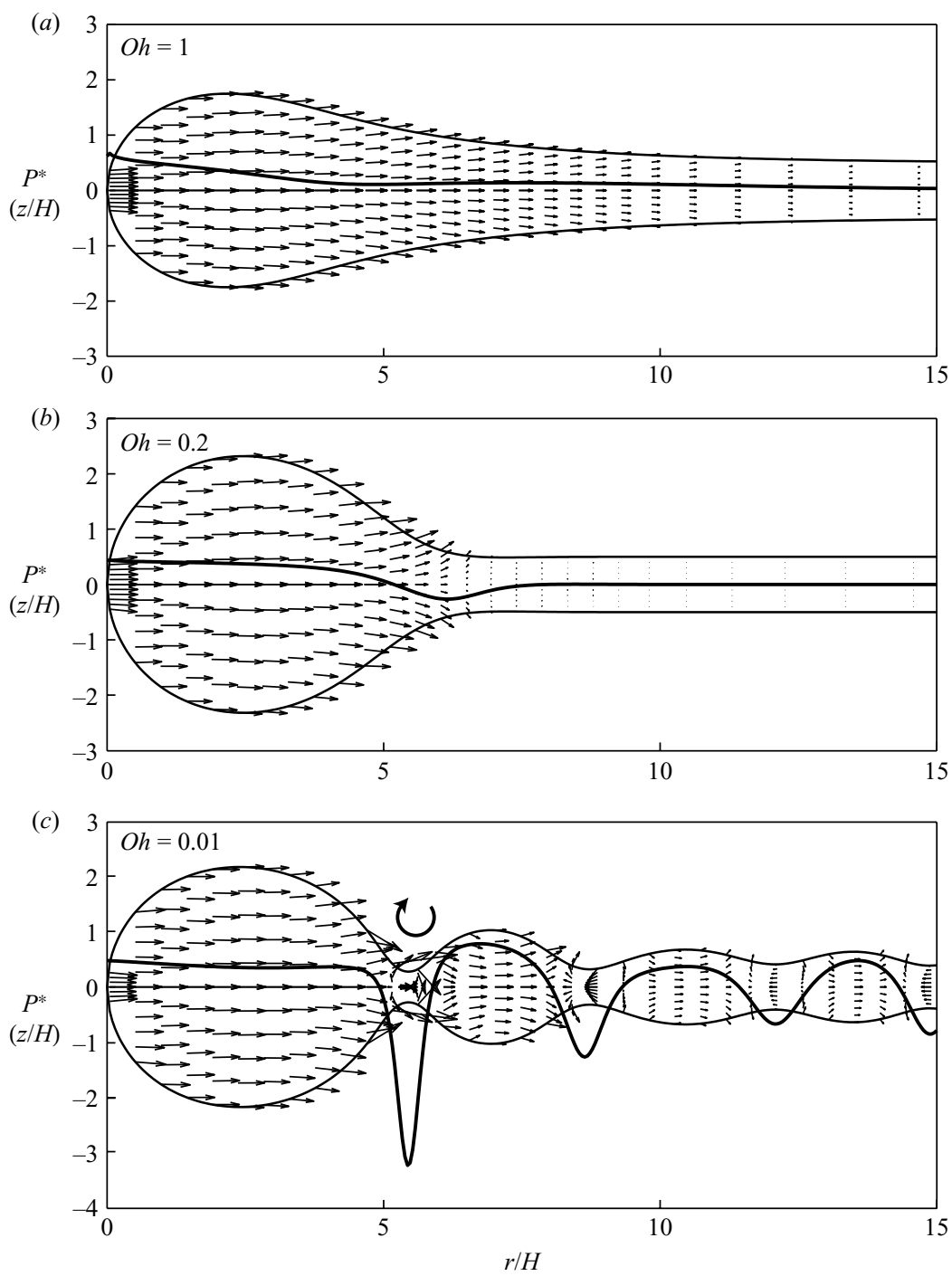

FIGURE 18. Velocity field and midplane pressure for $(a) O h=1.0,(b) O h=0.2$ and (c) $O h=0.01$. The velocity field is depicted by the arrows and the curve corresponds to the dimensionless pressure $P^{*}=H p / \gamma$ along the film midplane. For $O h=1(a)$, a monotonic decrease in the pressure prohibits the formation of a rim, which diffuses towards the bulk of the film. As $O h$ decreases, a rim begins to form due to the pressure drop at the rim neck $(b)$. For $O h=0.01$ $(c)$, vorticity generates capillary waves that are connected to the rim via a neck region. Note the pressure lows in the neck regions produced by the high local curvatures.

more violent at later times. Such increasing oscillations of the tip speed were also observed at the edge of a contracting filament in the simulations of Notz \& Basaran (2004) (figure 20). Depending on the Ohnesorge number, there were cases where the filament profiles they obtained were not single-valued functions of $r$ near pinch-off. As a caveat, we note that our lubrication model presumes a single-valued film profile and so cannot always accurately predict pinch-off times. Simulations at even lower Oh $(O h=0.005)$ reveal that sheet breakup can swiftly follow the initiation of sheet retraction. 


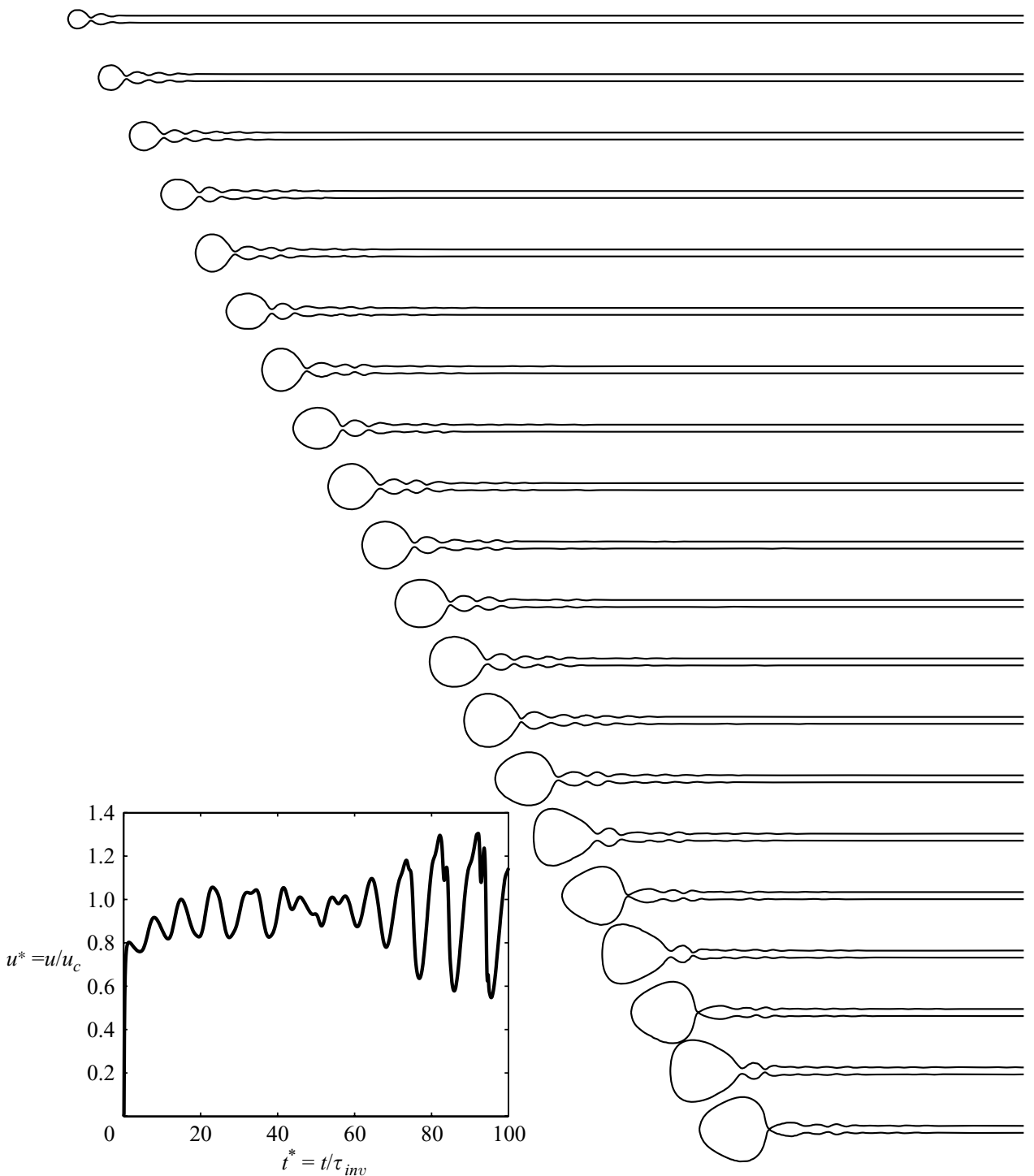

FIGURE 19. Sequence of the film profiles for $O h=0.01$ in time increments of $\delta t=5 t_{i n v}$; (inset) tip speed versus time. The capillary waves are more pronounced and begin to interact with the rim after an initial transient. This interaction becomes more violent as time progresses and eventually causes breakup. (Inset) The evolution of the tip speed resulting from the rim-wave interaction.

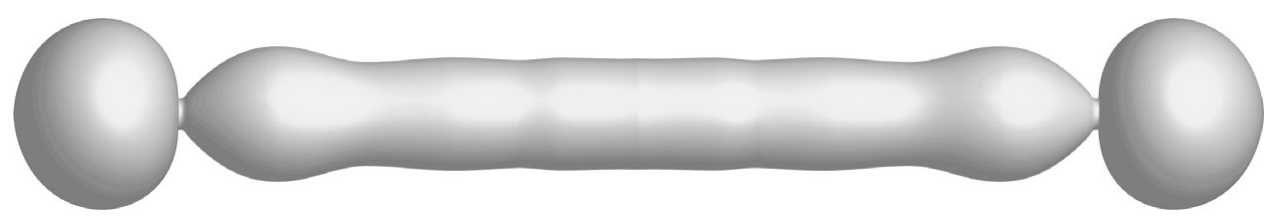

FIGURE 20. A contracting filament at $O h=0.001$ can exhibit similar breakup characteristics with a retracting sheet (reproduced from simulations of Notz \& Basaran 2004). 


\section{Discussion}

We have presented the results of a combined theoretical and numerical examination of the retraction of fluid sheets, giving particular attention to elucidating the influence of the sheet viscosity. First, we have clarified the shortcomings of the attempt of Dupré (1867) to use an energy principle to obtain the retraction speed, and corrected it by appropriate manipulation of the lubrication equations. In the planar geometry, half of the surface energy is converted to kinetic energy, and the other half dissipated through the action of viscosity, as was demonstrated numerically by Sünderhauf $e t$ al. (2002). We found that viscous forces do not contribute to the total momentum of the film; consequently our formulation yields the same long time limit deduced by Taylor (1959) and Culick (1960) from inviscid theory. This result can also be understood on the grounds that the viscous forces within the film are internal forces that do not contribute to the film's momentum budget. Most significantly, our study has yielded insight into the transient approach to the Taylor-Culick speed, a transient influenced by both geometry and viscosity.

In the low $\mathrm{Oh}$ regime $(\mathrm{Oh}<0.01)$, sheet retraction is inertia-dominated and capillary waves form ahead of a growing rim. For moderate $O h(0.01<O h<10)$, no capillary waves form ahead of the rim and for the high $O h(O h \gg 10)$ films, the retraction is dominated by viscosity and no rim forms. Brenner \& Gueyffier (1999) were the first to identify these regimes in the two-dimensional planar geometry. We have extended their work on retraction in this geometry by predicting analytically the dynamics in the high $\mathrm{Oh}$ regime at the onset of rupture. In particular, we deduced that the edge of the film recedes initially according to $t^{3 / 2}$, and that the maximum film thickness grows linearly in time. The retraction dynamics are markedly different in the circular geometry.

We have developed a lubrication model that allows us to investigate the retraction dynamics of a circular hole and made comparisons of our model with the experimental investigations of Debrégeas et al. (1995), Dalnoki-Veress et al. (1999) and Roth et al. (2005). Our direct manipulation of the lubrication equations to deduce the early stage dynamics in the high $\mathrm{Oh}$ limit provides an alternative to the energy argument presented by Debrégeas et al. (1995). Our results have also confirmed the conjecture of Brenner \& Gueyffier (1999) that geometry is important in sheet retraction especially in the high $O h$ regime. During the initial stages of retraction, the circular hole punctured on a sheet expands with a radial speed $\sim \mathrm{e}^{t / 2 \tau}$, while the planar sheet recedes with a speed $\sim \sqrt{t / \tau}$. This difference is associated with the contribution to the surface tension force coming from the azimuthal curvature that opposes the initial expansion of the circular hole and the viscous stresses that are relatively large when the punctured hole is small. In the long time limit the governing equations asymptote to those arising in the planar geometry and the rim speed approaches $u_{c}$.

We suggest that the discrepancy between the predicted and observed retraction rates in the study of Debrégeas et al. (1995) may be attributed in part to the details of the puncture, specifically the initial film profile. We have demonstrated that the initial retraction rate could be made faster with a slightly pointed initial film profile, but as time progresses, the retraction rate approaches that of a film with a nearly semi-circular initial profile. Similar observations were made by Roth et al. (2005) in their experiments on polystyrene films, but were not attributed to the film profile. It is hoped that our findings may motivate and inform further experiments on the early stages of film retraction.

Finally, we note that our model neglects any three-dimensional effects; specifically, variations in rim radius expected to accompany capillary instability of the rim (e.g. 
McEntee \& Mysels 1969; Pandit \& Davidson 1990). While rim instability is related to the Rayleigh-Plateau instability, the physical picture is more complex owing to rim growth. For instance in the moderate $O h$ regime, if a rim of radius $R$ were to pinch off due to the capillary instability, it would do so after a time $\tau_{c} \sim \sqrt{\rho R^{3} / 2 \gamma}$, which can be comparable to the appropriate time scale of retraction, $\tau_{i n v}=\sqrt{\rho H^{3} / 2 \gamma}$. This is especially true at the onset of retraction, where $H$ and $R$ are comparable. The tendency of the rim growth to suppress the onset of this instability was apparent in the experiments of McEntee \& Mysels (1969) and demonstrated in the numerical simulations (at $O h=0.1$ and 0.03) of Fullana \& Zaleski (1999), who suggested that the rim will eventually become unstable to perturbations with wavelengths that are typically a few orders of magnitude larger than the film thickness. The stability of the rim is enhanced not only by rim growth, but by the action of viscosity as was confirmed in the experiments of Debrégeas et al. (1995), Dalnoki-Veress et al. (1999) and Roth et al. (2005). Improved understanding of the rim stability will contribute to our ability to predict drop sizes resulting from film disintegration (see Bush \& Hasha 2004; Bremond \& Villermaux 2006; Roisman, Horvat \& Tropea 2006; Villermaux 2007).

John W. M. Bush gratefully acknowledges the financial support of the NSF through Career Grant CTS-0130465. The authors thank Jens Eggers for a number of valuable exchanges.

\section{Appendix. Numerical method}

In non-dimesionalizing the governing equations (3.15) and (3.16), the time scale is chosen according to the Ohnesorge number. By selecting the larger time scale of $\tau_{i n v}$ and $\tau_{v i s}$, we facilitate the computations since with the same computational cost, we can integrate the equations further in physical time. Since $\tau_{v i s}=O h \tau_{i n v}, \tau_{i n v}$ and $\tau_{v i s}$ are the appropriate time scales for, respectively, $O h<1$ and $O h>1$.

Equations (3.15) and (3.16) are rearranged to solve for $f=h^{2}$, because the slope of $h$, which goes like $\sqrt{r-r_{0}(t)}$ near the tip, becomes infinite there. Doing so avoids the difficulties in taking the derivatives of $h$ to evaluate the curvature at the tip, which now transforms to

$$
\kappa=8 \frac{2 f f_{r r}-f_{r}^{2}}{\left(16 f+f_{r}^{2}\right)^{3 / 2}}+\frac{f_{r}}{r\left(16 f+f_{r}^{2}\right)^{1 / 2}} .
$$

In their examination of jet breakup, Eggers \& Dupont (1994) remedied the difficulty of $h_{r}$ blowing up at the tip by fitting an even quartic polynomial to the jet profile in this region. Our approach is more natural in terms of implementation as the discretization of the equations is done without resorting to polynomial fits.

Since this is a free boundary problem, where the tip of the film moves, solving the system of equations requires the remeshing of the domain at each time step. To avoid this difficulty, we map the computational domain $\left(r^{\prime}, t^{\prime}\right)$ to the physical $(r, t)$-plane via the transformation

$$
\begin{aligned}
& r=\left(1-\frac{r_{0}(t)}{L}\right) r^{\prime}+r_{0}(t), \\
& t=t^{\prime},
\end{aligned}
$$

where $0 \leqslant r^{\prime} \leqslant L$ and $L$ is the radial extent of the film. Under this mapping, the edge of the film is always located at $r^{\prime}=0$ in the computational domain and $r=L$ 
is mapped to $r^{\prime}=L$; the free boundary problem is thus transformed into a fixed boundary problem. The derivatives must be transformed accordingly by

$$
\begin{aligned}
\frac{\partial}{\partial t} & =\frac{\partial}{\partial t^{\prime}}-\frac{1-r^{\prime} / L}{1-r_{0}(t) / L} u_{0}(t) \frac{\partial}{\partial x^{\prime}}, \\
\frac{\partial}{\partial x} & =\frac{1}{1-r_{0}(t) / L} \frac{\partial}{\partial x^{\prime}},
\end{aligned}
$$

where the tip speed

$$
u_{0}=\frac{\mathrm{d} r_{0}}{\mathrm{~d} t} .
$$

In Eggers' work, the failure of the lubrication approximation is remedied by neglecting the effect of viscosity near the tip; in the jet breakup problem, the condition at the jet tip is apparently not critical. Nevertheless, choosing the appropriate tip condition is crucial here and needs to be addressed more carefully. We see that when $\theta=\pi / 2$ and $f=0,(3.4 b)$ is trivially satisfied no matter what form $u_{2}$ takes. Hence, we infer $u_{2}$ by extrapolating over the values of $u_{2}$ in the interior, found using (3.13). Quadratic extrapolation requires

$$
\left.u_{2}\right|_{\text {tip }}=\frac{x_{1} x_{2}\left(x_{2}-x_{1}\right) u_{2,3}+x_{1} x_{3}\left(x_{1}-x_{3}\right) u_{2,2}+x_{2} x_{3}\left(x_{3}-x_{2}\right) u_{2,1}}{x_{1} x_{2}\left(x_{2}-x_{1}\right)+x_{1} x_{3}\left(x_{1}-x_{3}\right)+x_{2} x_{3}\left(x_{3}-x_{2}\right)},
$$

where $x_{i}=r_{i}-r_{0}$ is the $(i+1)$ th node in the computational grid at which $u_{2}$ takes the value $u_{2, i}$. Substituting $\theta=\pi / 2$ and $f=0$ in (3.4a) gives

$$
-\left.\frac{p}{\rho}\right|_{\text {tip }}=\frac{\gamma}{\rho} \kappa-2 v u_{0_{r}} .
$$

Using (A 6) in (3.10), we find that the velocity at the film edge evolves according to

$$
\frac{\mathrm{d} u_{0}}{\mathrm{~d} t}=\frac{\gamma}{\rho} \kappa_{r}+\left.2 v\left(-u_{0_{r r}}+\frac{u_{0_{r}}}{r}-\frac{u_{0}}{r^{2}}+u_{2}\right)\right|_{t i p} .
$$

The presence of the viscous term is essential here: its absence causes the hole to expand at a faster rate.

At the far end of the film $(r \rightarrow \infty)$, Neumann conditions are used for the thickness and velocity. As far as the initial film profile is concerned, it is imposed rather arbitrarily, by using a profile of the form

$$
\begin{aligned}
& f^{*}\left(r^{*}, 0 ; \alpha\right) \\
& \quad=1-\left(\frac{1}{2}-\alpha-r^{*}+R_{0}^{*}+\frac{1}{2} \sqrt{(1+2 \alpha)^{2}+4\left(r^{*}-R_{0}^{*}\right)\left(2 \alpha+r^{*}-R_{0}^{*}-1\right)}\right)^{2},
\end{aligned}
$$

where $\alpha$ is a parameter that controls the curvature profile and $r^{*} \geqslant R_{0}^{*}$. Equation (A 8) was constructed by perturbing trajectories on the phase plane of a particular dynamical system and essentially represents a smoothing of a semi-circular cap together with a rectangular strip. The smaller we take $\alpha$, the more pronounced the peak in the curvature gradient, and the finer the mesh required to resolve the sheet shape. In the numerical computations, we typically used $\alpha=1 / 20$ to $1 / 100$. As we increase $\alpha$, the tip curvature and its gradient increase according to

$$
\kappa\left(r=R_{0} ; \alpha\right)=-2-4 \alpha,
$$




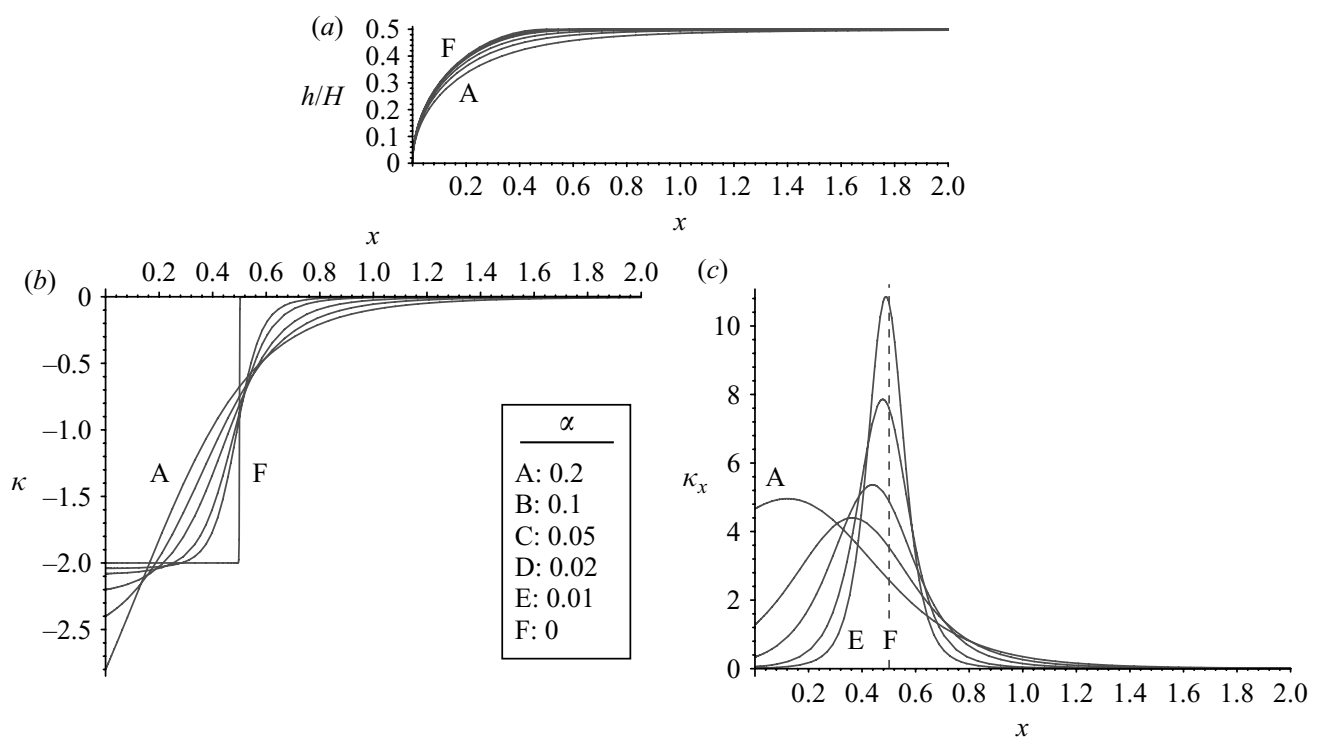

FIGURE 21. (a) Film profiles and their corresponding $(b)$ curvatures and $(c)$ curvature gradients for different values of $\alpha$. Curves A to $\mathrm{F}$ correspond to $\alpha=0.2,0.1,0.05,0.02,0.01$ and 0 , respectively.

$$
\kappa_{r}\left(r=R_{0} ; \alpha\right)=\frac{48 \alpha^{2}(3+2 \alpha)}{1+2 \alpha} .
$$

Figure 21 shows some representative plots of these profiles. Choosing such forms for the initial film profile allows us to study the effect of the initial tip curvature on the retraction dynamics by simply varying the parameter $\alpha$.

Following Eggers \& Dupont (1994), the numerical solution of (3.15) and (3.16) was implemented with a centred finite difference scheme on a staggered mesh over the interval $0 \leqslant r \leqslant L$. Implementation with a non-staggered grid introduced spurious oscillations in the curvature in the long time limit. Note that similar spurious oscillations occur in the pressure field, when the Navier-Stokes equations are solved by prescribing all velocities and pressures at the nodes of a Cartesian grid. Generally, we use a smoothly varying non-uniformly spaced mesh

$$
0=r_{1}<r_{2}<\cdots<r_{N}=L,
$$

and solve at each time step for $f_{i}$, the square of the thickness at $r=r_{i}$ and for $u_{i}$, the film speed $r=r_{i+1 / 2}=\left(r_{i}+r_{i+1}\right) / 2$ (see figure 22). Defining

$$
\begin{aligned}
\Delta r_{i} & =r_{i+1}-r_{i}, \\
\Delta r_{i+1 / 2} & =r_{i+1 / 2}-r_{i-1 / 2},
\end{aligned}
$$

we discretize the equation for $h_{i}$ (3.15) at each point $r_{i}$ and the equation for $u_{i}(3.16)$ at each point $r_{i+1 / 2}$. Doing so, we define

$$
\begin{aligned}
Q_{i}(\boldsymbol{h}, \boldsymbol{u})= & c\left[\frac{\Delta r_{i-1} u_{i}+\Delta r_{i} u_{i-1}}{\Delta r_{i}+\Delta r_{i-1}}-\left(1-\frac{r_{i}}{L}\right) u_{0}\right] \frac{f_{i+1}-f_{i-1}}{\Delta r_{i}+\Delta r_{i-1}} \\
& +2 f_{i} c\left(2 \frac{u_{i}-u_{i-1}}{\Delta r_{i}+\Delta r_{i-1}}+\frac{1}{r_{i}+r_{0} c} \frac{\Delta r_{i} u_{i-1}+\Delta r_{i-1} u_{i}}{\Delta r_{i}+\Delta r_{i-1}}\right),
\end{aligned}
$$




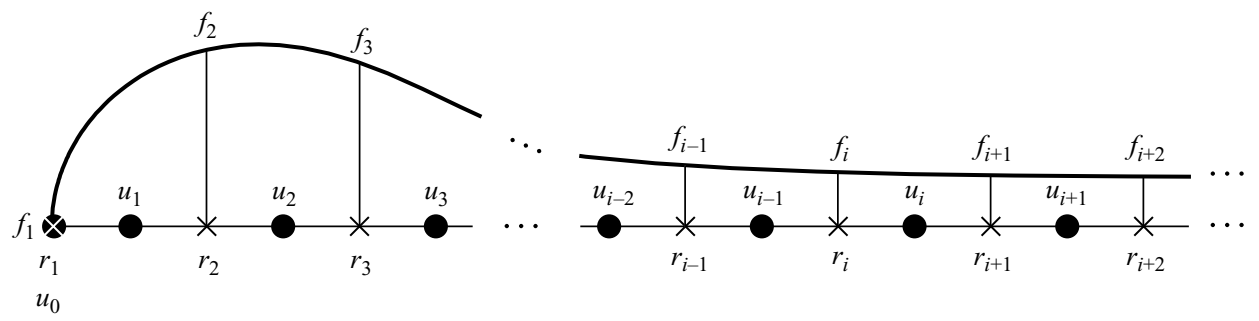

FigURE 22. The staggered grid used for the computation. The values of $f_{i}$ are prescribed at the mesh points $r_{i}$ and the values of $u_{i}$ are prescribed at $\left(r_{i}+r_{i+1}\right) / 2$.

$$
\begin{aligned}
W_{i}(\boldsymbol{h}, \boldsymbol{u})= & c\left[u_{i}-\left(1-\frac{r_{i+1 / 2}}{L}\right) u_{0}\right] \frac{u_{i+1}-u_{i-1}}{\Delta r_{i+3 / 2}+\Delta r_{i+1 / 2}}-\frac{1}{2} c \frac{\kappa_{i+1}-\kappa_{i}}{\Delta r_{i}} \\
& -\frac{8 O h c^{2}}{f_{i}+f_{i+1}}\left[\frac { 1 } { \Delta r _ { i } } \left(\frac{f_{i+1}}{r_{i+1}+r_{0} c} \frac{\left(r_{i+3 / 2}+r_{0} c\right) u_{i+1}-\left(r_{i+1 / 2}+r_{0} c\right) u_{i}}{\Delta r_{i+3 / 2}}\right.\right. \\
& \left.-\frac{f_{i}}{r_{i}+r_{0} c} \frac{\left(r_{i+1 / 2}+r_{0} c\right) u_{i}-\left(r_{i-1 / 2}+r_{0} c\right) u_{i-1}}{\Delta r_{i+1 / 2}}\right) \\
& \left.-\frac{f_{i+1}-f_{i}}{\Delta r_{i}}\left(\frac{1}{2} \frac{u_{i+1}-u_{i-1}}{\Delta r_{i+3 / 2}+\Delta r_{i+1 / 2}}+\frac{3 u_{i}}{4\left(r_{i+1 / 2}+r_{0} c\right)}\right)\right]
\end{aligned}
$$

where $c(t)=1 /\left(1-r_{0}(t) / L\right)$ and the curvatures $\kappa_{i}$ are formed as the usual secondorder centred finite differences, defined at the mesh points $r_{i}$. Time integration was performed with the implicit $\theta$-weighted finite difference scheme

$$
\begin{aligned}
& \frac{f_{i}^{t}-f_{i}^{t-\delta t}}{\delta t}+\theta Q_{i}\left(\boldsymbol{h}^{t}, \boldsymbol{u}^{t}\right)+(1-\theta) Q_{i}\left(\boldsymbol{h}^{t-\delta t}, \boldsymbol{u}^{t-\delta t}\right)=0, \\
& \frac{u_{i}^{t}-u_{i}^{t-\delta t}}{\delta t}+\theta W_{i}\left(\boldsymbol{h}^{t}, \boldsymbol{u}^{t}\right)+(1-\theta) W_{i}\left(\boldsymbol{h}^{t-\delta t}, \boldsymbol{u}^{t-\delta t}\right)=0
\end{aligned}
$$

where $\boldsymbol{h}^{t}$ and $\boldsymbol{u}^{t}$ correspond to the solution vectors at time $t$, and $\delta t$ is the time step of the computation. In order to solve resulting system (A 16), we use a matrixfree Newton-Krylov method which is presented in more detail in Savva (2007); an overview of Jacobian-free methods can be found in the paper by Knoll \& Keyes (2004) and further details can be sought in numerical analysis texts (see for example Kelley 1995, 2003).

\section{REFERENCES}

Batchelor, G. K. 1967 An Introduction to Fluid Mechanics. Cambridge University Press. BaYvel, L. \& Orzechowski, Z. 1993 Liquid Atomization. Taylor-Francis.

Bremond, N. \& Villermaux, E. 2006 Atomization by jet impact. J. Fluid Mech. 549, 273-306.

Brenner, M. P. \& Gueyffier, D. 1999 On the bursting of viscous sheets. Phys. Fluids 11, 737-739. Buguin, A., Vovelle, L. \& Brochard-Wyart, F. 1999 Shocks in inertial dewetting. Phys. Rev. Lett. 83, 1183-1186.

Bush, J. W. M. \& Hasha, A. E. 2004 On the collision of laminar jets: fluid chains and fishbones. J. Fluid Mech. 511, 285-310.

Chepushtanova, S. V. \& Kliakhandler, I. L. 2007 Slow rupture of viscous films between parallel needles. J. Fluid Mech. 573, 297-310.

Culick, F. E. C. 1960 Comments on a ruptured soap film. J. Appl. Phys. 31, 1128-1129. 
Dalnoki-Veress, K., Nickel, B. G., Roth, C. \& Dutcher, J. R. 1999 Hole formation and growth in freely standing polystyrene films. J. Fluid Mech. 59 (2), 2153-2156.

Debrégeas, G., De GenNes, G. P. \& Brochard-Wyart, F. 1998 The life and death of viscous "bare" bubbles. Science 279, 1704-1707.

Debrégeas, G., Martin, P. \& Brochard-Wyart, F. 1995 Viscous bursting of suspended films. Phys. Rev. Lett. 75, 3886-3889.

Deyrail, Y., Mesri, Z. El, Huneault, M., Zeghloul, A. \& Bousmina, M. 2007 Analysis of morphology development in immiscible newtonian polymer mixtures during shear flow. J. Rheol. 51, 781-797.

Dupré, M. A. 1867 Sixième memoire sur la theorie méchanique de la chaleur. Ann. Chim. Phys. 4 (11), 194-220.

Eggers, J. \& Brenner, M. P. 2000 Spinning jets. In Proceedings of the IUTAM Symposium on Nonlinear Waves in Multi-Phase Flow (ed. H.-C. Chang), pp. 185-194. Kluwer.

EgGers, J. \& Dupont, T. F. 1994 Drop formation in a one-dimensional approximation of the Navier-Stokes equation. J. Fluid Mech. 262, 205-221.

Fullana, G. M. \& ZALESKI, S. 1999 Stability of a growing rim in a liquid sheet of uniform thickness. Phys. Fluids 11, 952-954.

de Gennes, P. G., Brochart-Wyart, F. \& Quéré, D. 2003 Capillarity and Wetting Phenomena: Drops, Bubbles, Pearls, Waves. Springer.

Keller, J. B. 1983 Breaking of liquid films and threads. Phys. Fluids 26, 3451-3453.

Keller, J. B. \& Miksis, M. J. 1983 Surface tension driven flows. SIAM J. Appl. Math. 43, 268-277.

Kelley, C. T. 1995 Iterative Methods for Linear and Nonlinear Equations. SIAM.

Kelley, C. T. 2003 Solving Nonlinear Equations with Newton's Method. SIAM.

KNoll, D. A. \& Keyes, D. E. 2004 Jacobian-free Newton-Krylov methods: a survey of approaches and applications. J. Comp. Phys. 193, 357-397.

Lefebvre, A. H. 1989 Liquid Atomization. Hemisphere.

McEntee, W. R. \& Mysels, K. J. 1969 The bursting of soap films. I. An experimental study. J. Phys. Chem. 73, 3018-3028.

Miyamoto, K. \& KatagiRi, Y. 1997 Curtain coating. In Liquid Film Coating (ed. S. F. Kistler \& P. M. Schweizer), pp. 463-494. Chapman-Hall.

Notz, P. K. \& Basaran, O. A. 2004 Dynamics and breakup of contracting filaments. J. Fluid Mech. 512, 223-256.

Pandit, A. B. \& Davidson, J. F. 1990 Hydrodynamics of the rupture of thin liquid films. J. Fluid Mech. 212, 11-24.

Pomeau, Y. \& Villermaux, E. 2006 Two hundred years of capillarity research. Phys. Today 59, $39-44$.

Ranz, W. E. 1950 Some experiments on the dynamics of liquid films. J. Appl. Phys. 30, 1950-1955.

RAYLEIGH, LORD 1891 Some applications of photography. Nature 44, 249-254.

Roisman, I. V., Horvat, K. \& Tropea, C. 2006 Spray impact: rim transverse instability initiating fingering and splash, and description of secondary spray. Phys. Fluids 18, 102104.

Roth, C. B., Deh, B., Nickel, B. G. \& Dutcher, J. R. 2005 Evidence of convective constraint release during hole growth in freely standing polystyrene films at low temperatures. Phys. Rev. E 72 (2), 021802.

Savva, N. 2007 Viscous fluid sheets. PhD thesis, Massachusetts Institute of Technology.

Song, M. \& Tryggvason, G. 1999 The formation of thick borders on an initially stationary fluid sheet. Phys. Fluids 11, 2487-2493.

Sünderhauf, G., Raszillier, H. \& Durst, F. 2002 The retraction of the edge of a planar liquid sheet. Phys. Fluids 14, 198-208.

TAYLOR, G. I. 1959 The dynamics of thin sheets of fluid. III. Disintegration of fluid sheets. Proc. R. Soc. Lond. Ser. A 253, 313-321.

TAYlor, G. I. \& Michael, D. I. 1973 On making holes in a sheet of fluid. J. Fluid Mech. 58, 625-639.

Villermaux, E. 2007 Fragmentation. Annu. Rev. Fluid Mech. 39, 419-446. 